\title{
On Time-Dependent Functionals of Diffusions Corresponding to Divergence Form Operators
}

\author{
Tomasz Klimsiak
}

Received: 18 January 2011 / Revised: 3 August 2011 / Published online: 28 September 2011

(C) The Author(s) 2011. This article is published with open access at Springerlink.com

\begin{abstract}
We consider processes of the form $[s, T] \ni t \mapsto u\left(t, X_{t}\right)$, where $\left(X, P_{s, x}\right)$ is a multidimensional diffusion corresponding to a uniformly elliptic divergence form operator. We show that if $u \in \mathbb{L}_{2}\left(0, T ; H_{\rho}^{1}\right)$ with $\frac{\partial u}{\partial t} \in \mathbb{L}_{2}\left(0, T ; H_{\rho}^{-1}\right)$ then there is a quasi-continuous version $\tilde{u}$ of $u$ such that $\tilde{u}\left(t, X_{t}\right)$ is a $P_{s, x}$-Dirichlet process for quasi-every $(s, x) \in[0, T) \times \mathbb{R}^{d}$ with respect to parabolic capacity, and we describe the martingale and the zero-quadratic variation parts of its decomposition. We also give conditions on $u$ ensuring that $\tilde{u}\left(t, X_{t}\right)$ is a semimartingale.
\end{abstract}

Keywords Dirichlet process · Diffusion · Divergence form operator

Mathematics Subject Classification (2000) Primary 60H05 · Secondary 60H30

\section{Introduction}

In the present paper, we study the structure of additive functionals (AFs for short) of the form $X^{u}=\left\{X_{s, t}^{u} \equiv u\left(t, X_{t}\right)-u\left(s, X_{s}\right) ; 0 \leq s \leq t \leq T\right\}$, where $u: Q_{T} \equiv[0, T] \times$ $\mathbb{R}^{d} \rightarrow \mathbb{R}$ and $\mathbb{X}=\left\{\left(X, P_{s, x}\right) ;(s, x) \in Q_{T}\right\}$ is a Markov family corresponding to the operator

$$
L_{t}=\frac{1}{2} \sum_{i, j=1}^{d} \frac{\partial}{\partial x_{i}}\left(a_{i j} \frac{\partial}{\partial x_{j}}\right)+\sum_{i=1}^{d} b_{i} \frac{\partial}{\partial x_{i}}
$$

T. Klimsiak (凶)

Faculty of Mathematics and Computer Science, Nicolaus Copernicus University, Chopina 12/18, 87-100 Torun, Poland

e-mail: tomas@mat.uni.torun.pl 
with measurable coefficients $a: Q_{T} \rightarrow \mathbb{R}^{d} \otimes \mathbb{R}^{d}, b: Q_{T} \rightarrow \mathbb{R}^{d}$ such that

$$
\lambda|\xi|^{2} \leq \sum_{i, j=1}^{d} a_{i j}(t, x) \xi_{i} \xi_{j} \leq \Lambda|\xi|^{2}, \quad a_{i j}=a_{j i}, \quad\left|b_{i}(t, x)\right| \leq \Lambda_{1}, \quad \xi \in \mathbb{R}^{d}
$$

for some $0<\lambda \leq \Lambda$ and $\Lambda_{1}>0$ (see [26, 29, 37]).

It is known (see $[22,32]$ ) that for every $(s, x) \in Q_{\hat{T}} \equiv[0, T) \times \mathbb{R}^{d}$ the process $X_{s, .} \equiv X .-X_{s}$ is under $P_{s, x}$ a continuous Dirichlet process on $[s, T]$ in the sense of Föllmer [14]. In the paper, we first develop some stochastic calculus for timedependent functionals of $\mathbb{X}$. Secondly, we give mild regularity conditions on $u$ under which the functional $X_{s, \text {. }}^{u}$ is a Dirichlet process under $P_{s, x}$ and, if this is the case, we describe the martingale part $M^{u}$ and the zero-quadratic variation part $A^{u}$ of its decomposition

$$
X_{s, t}^{u}=M_{s, t}^{u}+A_{s, t}^{u}, \quad t \in[s, T], P_{s, x} \text {-a.s. }
$$

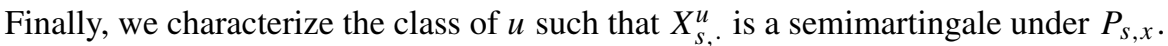

It is known that general Dirichlet processes are stable under $C^{1}$ transformations (see $[4,9]$ ). $C^{1}$-regularity of $u$ is too strong in applications we have in mind. Our main motivation to investigate functionals of the form $X^{u}$ comes from the fact that they appear in probabilistic analysis of strong solutions to parabolic PDEs or variational inequalities involving the operator $L_{t}$ (see [20, 33, 34]). Therefore, the natural assumption on $u$ is that it belongs to some Sobolev space and in general is even not continuous.

Time-independent functionals of time-homogeneous diffusions are quite well investigated. Let $\mathcal{X}$ be a locally compact separable metric space and let $m$ be a positive Radon measure on $\mathcal{X}$ such that $\operatorname{supp}[m]=\mathcal{X}$. Let $\left\{\left(X, P_{X}\right) ; x \in \mathcal{X}\right\}$ be an $m$ symmetric Hunt process with Dirichlet form $(\mathcal{E}, D(\mathcal{E}))$ on $\mathbb{L}_{2}(\mathcal{X}, m)$. It is known (see [18]) that for every $u \in D(\mathcal{E})$ there exists an $\mathcal{E}$-quasi-continuous version of $u$ (still denoted by $u$ ) such that $X^{u}$ admits the so-called Fukushima decomposition, i.e.,

$$
X_{t}^{u}=M_{t}^{u}+A_{t}^{u}, \quad t \in[0, T], P_{x} \text {-a.s. }
$$

for $\mathcal{E}$-q.e. $x \in \mathcal{X}$, where $M^{u}$ is a continuous martingale $\mathrm{AF}$ of finite energy and $A^{u}$ is a continuous AF of zero energy. A simple calculation (see [18, p. 201]) shows that $A^{u}$ has zero-quadratic variation on $[0, T]$ under the measure $P_{v}(\cdot)=\int_{\mathcal{X}} P_{x}(\cdot) d v(x)$ along dyadic partitions of $[0, T]$ for every Radon measure $v \ll m$. Hence, to prove that $X^{u}$ is a Dirichlet process in the sense of Föllmer, one should weaken the assumption on the absolute continuity of $v$ and on the sequence of partitions. In [12], the authors weakened the assumption on the starting measures $v$ in the case of Dirichlet form $(\mathcal{E}, D(\mathcal{E}))$ on $\mathbb{L}_{2}\left(\mathbb{R}^{d}, m\right)$ with the Lebesgue measure $m$, defined by

$$
\mathcal{E}(u, v)=\frac{1}{2}\langle a \nabla u, \nabla v\rangle_{2}, \quad u, v \in D(\mathcal{E})=H^{1}\left(\mathbb{R}^{d}\right),
$$

where $a(t, x)=a(x), x \in \mathbb{R}^{d}$. The class of measures considered in [12] includes in particular the Dirac measure $\delta_{\{x\}}$ for $\mathcal{E}$-q.e. $x \in \mathbb{R}^{d}$, which shows that $X^{u}$ is a Dirichlet process on $[0, T]$ under $P_{x}$ for $\mathcal{E}$-q.e. $x \in \mathbb{R}^{d}$ along dyadic partitions. It is 
worth mentioning that in the case of non-symmetric diffusions the approach of [12] breaks down.

A different approach to the problem of investigating $X^{u}$ in case

$$
\mathcal{E}(u, v)=\frac{1}{2}\langle a \nabla u, \nabla v\rangle_{2}+\langle b \nabla u, v\rangle_{2}, \quad u, v \in D(\mathcal{E})=H^{1}\left(\mathbb{R}^{d}\right),
$$

with $a(t, x)=a(x), b(t, x)=b(x)$ was adopted in [31]. In [31], it is shown that if $u \in W_{q}^{1}\left(\mathbb{R}^{d}\right)$ with $q>2$ then $X^{u}$ is a continuous Dirichlet process in the sense of Föllmer for $\mathcal{E}$-q.e. $x \in \mathbb{R}^{d}$ (see also [29, 32] where time-inhomogeneous diffusions are also considered).

In the case of a one-dimensional Wiener process $W$, it is known (see [16]) that $W^{u}$ is a continuous Dirichlet process in the sense of Föllmer for every starting point $x \in \mathbb{R}$ if $u \in H^{1}(\mathbb{R})$, and it appears that this condition is necessary (see [7]). In the case of a multidimensional Wiener process, one can deduce from [15] that $W^{u}$ is a continuous Dirichlet process in the sense of Föllmer on $[0, T]$ for q.e. starting points $x \in \mathbb{R}^{d}$ if $u \in H^{1}\left(\mathbb{R}^{d}\right)$.

To our knowledge, in the case where $u$ depends on time, only few results are available. In [22], diffusions corresponding to $L_{t}$ are considered. It is shown there that $X^{u}$ is a continuous Dirichlet process on $[s, T]$ in the sense of Föllmer for every $(s, x) \in Q_{\hat{T}}$ if $\sup _{t \in[0, T]}\left(\|\nabla u(t)\|_{p}+\left\|\frac{\partial u}{\partial t}(t)\right\|_{p}\right)<\infty$ for some $p>d \wedge 2$. In [8], necessary and sufficient conditions on $u$ for $X^{u}$ to be a semimartingale are given in the case where $X$ is a one-dimensional Wiener process.

We will now briefly describe the content of the paper. As already mentioned, we are interested in solutions to parabolic PDEs or parabolic variational inequalities involving $L_{t}$. Therefore, our basic assumption on $u$ is that $u \in \mathcal{W}_{\rho}$, where $\mathcal{W}_{\rho}=\{u \in$ $\left.\mathbb{L}_{2}\left(0, T ; H_{\rho}^{1}\right) ; \frac{\partial u}{\partial t} \in \mathbb{L}_{2}\left(0, T ; H_{\rho}^{-1}\right)\right\}$ ( $\rho$ is some weight), i.e., $u$ belongs to the natural space for strong solutions of such problems. Let $\operatorname{cap}_{L}: 2^{Q_{\hat{T}}} \rightarrow \mathbb{R}^{+} \cup\{+\infty\}$ be the parabolic capacity associated with $L_{t}$ (see [28]) or, equivalently, a restriction to $Q_{\hat{T}}$ of the capacity generated by the time-dependent Dirichlet form

$$
\mathcal{E}(u, v)= \begin{cases}\int_{\mathbb{R}} \mathcal{E}^{(t)}(u(t), v(t)) d t-\int_{\mathbb{R}}\left\langle\frac{\partial u}{\partial t}(t), v(t)\right\rangle, & u \in \mathcal{W}, v \in \mathbb{L}_{2}\left(\mathbb{R} ; H^{1}\right), \\ \int_{\mathbb{R}} \mathcal{E}^{(t)}(u(t), v(t)) d t+\int_{\mathbb{R}}\left\langle\frac{\partial v}{\partial t}(t), u(t)\right\rangle, & v \in \mathcal{W}, u \in \mathbb{L}_{2}\left(\mathbb{R} ; H^{1}\right),\end{cases}
$$

where $\mathcal{W}$ denotes $\mathcal{W}_{\rho}$ with $\rho \equiv 1$,

$$
\mathcal{E}^{(t)}(u, v)=\frac{1}{2}\langle a(t) \nabla u, \nabla v\rangle_{2}+\langle b(t) \nabla u, v\rangle_{2}, \quad t \in[0, T],
$$

$\mathcal{E}^{(t)}(u, v)=\mathcal{E}^{(0)}(u, v)$ for $t \leq 0$ and $\mathcal{E}^{(t)}(u, v)=\mathcal{E}^{(T)}(u, v)$ for $t \geq T$. In the paper, we provide various conditions on $u$ ensuring that for cap $_{L}$-quasi every (q.e. for short) $(s, x) \in Q_{\hat{T}}$ the process $X_{s, \cdot}^{u}$ is under $P_{s, x}$ a continuous Dirichlet process on $[s, T]$ in the sense of Föllmer or is a continuous semimartingale.

For the convenience of the reader, we begin in Sect. 2 with basic information on various definitions of parabolic capacity associated with $L_{t}$.

In Sect. 3, we formulate Fukushima's and Lyons-Zheng's decomposition of $X$ under $P_{s, x}$. Using the latter decomposition, we investigate additive functionals of 
the form $\int \operatorname{div} \bar{f}\left(\theta, X_{\theta}\right) d \theta$, where $\operatorname{div} \bar{f}$ stands for the divergence of the vector field $\bar{f}=\left(f^{1}, \ldots, f^{d}\right)$ such that $f^{i} \in \mathbb{L}_{2}^{\text {loc }}\left(Q_{T}\right), i=1, \ldots, d$. It is known that in the case of time-homogeneous diffusions $\left\{\left(X, P_{x}\right) ; x \in \mathbb{R}^{d}\right\}$ corresponding to $L_{t}$ with timeindependent coefficients, such functionals may be defined under the measure $P_{m}$ as a forward-backward integral with respect to martingales from the Lyons-Zheng decomposition of $X^{u}$ (see [36]). We show that the functionals can be well defined for time-inhomogeneous diffusions and what is more important, under the measure $P_{s, x}$ for q.e. $(s, x) \in Q_{\hat{T}}$ (see [34] for similar results). We also show that if $u \in H^{1}\left(\mathbb{R}^{d}\right)$ then under some additional regularity conditions on the coefficient $a, X^{u}$ is a continuous Dirichlet process in the sense of Föllmer under $P_{x}$ for $\mathcal{E}$-q.e. $x \in \mathbb{R}^{d}$, where $\mathcal{E}$ is given by (1.4).

In Sect. 4, we show that each $u \in \mathcal{W}_{\rho}$ has a quasi-continuous version, still denoted

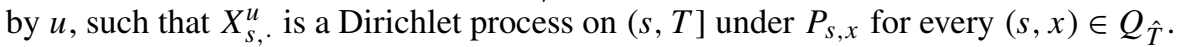
Under mild additional regularity conditions on $u$, it is a Dirichlet process on $[s, T]$ for cap $_{L}$-q.e. $(s, x) \in Q_{\hat{T}}$. We also describe the martingale and the zero-quadratic variation parts of the decomposition (1.3) and show that (1.3) implies the Fukushima decomposition of $X^{u}$ into martingale AF of finite energy and CAF of zero energy.

In Sect. 5, we introduce the definition of the integral with respect to continuous additive functionals (CAFs for short) of $\mathbb{X}$ of zero-quadratic variation associated with functionals in $\mathbb{L}_{2}\left(0, T ; H_{\rho}^{-1}\right)$. The key result here says that given such a CAF $A$ and a bounded $\eta \in \mathcal{W}_{\rho}$ one can find a sequence $\left\{A^{n}\right\}$ of square-integrable CAFs of finite variation such that for q.e. $(s, x) \in Q_{\hat{T}}$,

$$
E_{s, x} \sup _{s \leq t \leq T}\left|\int_{s}^{t} \eta\left(\theta, X_{\theta}\right) d A_{s, \theta}^{n}-\int_{s}^{t} \eta\left(\theta, X_{\theta}\right) d A_{s, \theta}\right| \rightarrow 0 .
$$

This approximation result enables us to handle integrals with respect to CAFs corresponding to functionals in $\mathbb{L}_{2}\left(0, T ; H_{\rho}^{-1}\right)$. As a first application, we show that such CAFs are uniquely determined by their Laplace transforms.

In Sect. 6, we are concerned with the problem of finding minimal conditions on $u \in \mathcal{W}_{\rho}$ under which $X^{u}$ is a semimartingale. Our main result proven here says that $X_{s}^{u}$, is a locally finite semimartingale under $P_{s, x}$ for q.e. $(s, x) \in Q_{\hat{T}}$ if and only if $\left(\frac{\partial}{\partial t}+L_{t}\right) u$ is a signed Radon measure.

Finally, in Sect. 6 we collect some useful estimates for diffusions $\mathbb{X}$ and related estimates on the fundamental solution $p$ and weak solutions of the Cauchy problem associated with $L_{t}$.

In the paper, we will use the following notation:

$$
\begin{aligned}
& Q_{s t}=[s, t] \times \mathbb{R}^{d}, \quad Q_{t}=[0, t] \times \mathbb{R}^{d}, \quad Q_{\hat{T}}=[0, T) \times \mathbb{R}^{d}, \\
& \nabla=\left(\frac{\partial}{\partial x_{1}}, \ldots, \frac{\partial}{\partial x_{d}}\right) .
\end{aligned}
$$

$C_{c}\left(Q_{T}\right)\left(C_{c}\left(\mathbb{R}^{d}\right)\right)$ is the space of all continuous functions with compact support in $Q_{T}$ (in $\mathbb{R}^{d}$ ).

$\mathbb{L}_{p}\left(\mathbb{R}^{d}\right)$ is the usual Banach space of measurable functions on $\mathbb{R}^{d}$ with the norm $\|u\|_{p}=\left(\int_{\mathbb{R}^{d}}|u(x)|^{p} d x\right)^{1 / p}, \mathbb{L}_{p, q}\left(Q_{t T}\right)$ is the Banach space of measurable functions 
on $Q_{t T}$ with the norm $\|u\|_{p, q, t, T}=\left(\int_{t}^{T}\left(\int_{\mathbb{R}^{d}}|u(s, x)|^{p} d x\right)^{p / q} d s\right)^{1 / q}, \mathbb{L}_{p}\left(Q_{t T}\right)=$ $\mathbb{L}_{p, p}\left(Q_{t T}\right),\|u\|_{p, p, t, T}=\|u\|_{p, t, T}$, and $\|u\|_{p, T}=\|u\|_{p, 0, T}$.

Let $\rho \in \mathcal{R}_{I}$. By $\mathbb{L}_{p, \rho}\left(\mathbb{R}^{d}\right)\left(\mathbb{L}_{p, q, \rho}\left(Q_{t T}\right)\right)$ we denote the space of functions $u$ such that $u \rho \in \mathbb{L}_{p}\left(\mathbb{R}^{d}\right)\left(u \rho \in \mathbb{L}_{p, q}\left(Q_{t, T}\right)\right)$ equipped with the norm $\|u\|_{p, \rho}=\|u \rho\|_{p}$ $\left(\|u\|_{p, q, \rho, t, T}=\|u \rho\|_{p, q, t, T}\right)$. We write $K \subset \subset X$ if $K$ is a compact subset of $X$. By $\langle\cdot, \cdot\rangle_{2}$ we denote the usual inner product in $\mathbb{L}_{2}\left(\mathbb{R}^{d}\right)$ and by $\langle\cdot, \cdot\rangle_{2, \rho}$ the inner product in $\mathbb{L}_{2, \rho}\left(\mathbb{R}^{d}\right)$.

$W_{p, \rho}^{1}$ is the Banach space consisting of all elements $u$ of $\mathbb{L}_{p, \rho}\left(\mathbb{R}^{d}\right)$ having generalized derivatives $\frac{\partial u}{\partial x_{i}}, i=1, \ldots, d$, in $\mathbb{L}_{p, \rho}\left(\mathbb{R}^{d}\right)$ with the dual space $W_{p, \rho}^{-1}$. We denote $H_{\rho}^{1}=W_{2, \rho}^{1} . \mathcal{W}_{\rho}$ is the subspace of $\mathbb{L}_{2}\left(0, T ; H_{\rho}^{1}\right)$ consisting of all elements $u$ such that $\frac{\partial u}{\partial t} \in \mathbb{L}_{2}\left(0, T ; H_{\rho}^{-1}\right)$, where $H_{\rho}^{-1}$ is the dual space to $H_{\rho}^{1}$ (see [23] for details). By $\langle\cdot, \cdot\rangle_{\rho}$ we denote the duality pairing between spaces $H_{\rho}^{1}, H_{\rho}^{-1}$ and by $\|\cdot\|_{*}$ we denote the norm in Banach space $\mathbb{L}_{2}\left(0, T ; H_{\rho}^{-1}\right)$.

By $\bar{f}$ we denote the vector function $\left(f^{1}, \ldots, f^{d}\right)$. We write $\bar{f} \in \mathbb{L}_{2, \rho}\left(Q_{T}\right)$ if $f^{i} \in \mathbb{L}_{2, \rho}\left(Q_{T}\right), i=1, \ldots, d$. By $\mathcal{M}\left(\mathcal{M}^{+}\right)$we denote the set of all Radon measures (positive Radon measures) on $Q_{T}$, and by $\mathcal{M}^{+}([0, T])$ the set of positive Radon measures on $[0, T]$. $\mathcal{B}(E)\left(\mathcal{B}_{b}(E), \mathcal{B}_{b}^{\text {loc }}(E), \mathcal{B}^{+}(E)\right)$ denotes the set of all Borel (bounded, locally bounded, positive) real functions on a topological space $E$.

By $C$ we denote a general constant which may vary from line to line, but depends only on fixed parameters.

\section{Parabolic Capacity}

Let $d \geq 1$ and let $\mathcal{R}$ denote the space of all functions $\rho: \mathbb{R}^{d} \rightarrow \mathbb{R}$ of the form $\rho(x)=$ $\left(1+|x|^{2}\right)^{-\alpha}, x \in \mathbb{R}^{d}$, for some $\alpha \in \mathbb{R}$, and let $\mathcal{R}_{I}$ be the space of all $\rho \in \mathcal{R}$ such that $\int_{\mathbb{R}^{d}} \rho(x) d x<\infty$. Unless otherwise stated, in the sequel we will always assume that $\rho \in \mathcal{R}_{I}$. We also write $\rho_{x}(y)=\rho(y-x), y \in \mathbb{R}^{d}$.

Let $\Phi \in \mathbb{L}_{2}\left(0, T ; H_{\rho}^{-1}\right)$. It is well known that $\Phi$ admits the decomposition $\Phi=f^{0}+\operatorname{div} \bar{f}$ for some $f^{0}, \bar{f} \in \mathbb{L}_{2, \rho}\left(Q_{T}\right)$, i.e., $\Phi(\eta)=\left\langle f^{0}, \eta\right\rangle_{2, \rho}-\left\langle\bar{f}, \nabla\left(\rho^{2} \eta\right)\right\rangle_{2}$. This decomposition is not unique, but it is known that for every such decomposition $\|\Phi\|_{*} \leq\left\|f^{0}\right\|_{2, \rho, T}+\|\bar{f}\|_{2, \rho, T}$ and there exists a pair which realizes the norm. If, in addition, $\Phi \geq 0$, i.e., $\Phi(\eta) \geq 0$ for any positive $\eta \in \mathbb{L}_{2}\left(0, T ; H_{\rho}^{1}\right)$, then by Riesz's theorem there is a Radon measure $\mu$ on $Q_{T}$ such that

$$
\Phi(\eta)=\int_{Q_{T}} \eta d \mu
$$

for every $\eta \in C_{0}^{\infty}\left(Q_{T}\right)$. Let us observe that

$$
\mu\left(\{t\} \times \mathbb{R}^{d}\right)=0, \quad t \in[0, T] .
$$

Indeed, if $\left\{\eta_{n}\right\} \subset C_{0}^{\infty}\left(Q_{T}\right)$ is a sequence of positive functions such that $\eta_{n} \downarrow \mathbf{1}_{\{t\} \times \mathbb{R}^{d}}$ pointwise and in $\mathbb{L}_{2}\left(0, T ; H_{\rho}^{1}\right)$, then

$$
0=\Phi(\eta)=\lim _{n \rightarrow \infty} \Phi\left(\eta_{n}\right)=\lim _{n \rightarrow \infty} \int_{\check{Q}_{T}} \eta_{n} d \mu=\mu\left(\{t\} \times \mathbb{R}^{d}\right) .
$$


Let us define the capacity of $E \subset \subset \check{Q}_{T} \equiv(0, T) \times \mathbb{R}^{d}$ by

$$
\begin{gathered}
\overline{\operatorname{cap}}_{\check{Q}_{T}}(E)=\inf \left\{\int_{Q_{T}}|\nabla \eta(t, x)|^{2} d t d x+\int_{Q_{T}}|\eta(t, x)|^{2} d t d x:\right. \\
\left.\eta \in C_{0}^{\infty}\left(\check{Q}_{T}\right), \eta \geq \mathbf{1}_{E}\right\} .
\end{gathered}
$$

The capacity can be extended in a standard way to the Borel $\sigma$-field $\mathcal{B}\left(\check{Q}_{T}\right)$ of subsets of $\check{Q}_{T}$. For $E \subset \subset \check{Q}_{T}$ and $\eta \in C_{0}^{\infty}\left(\check{Q}_{T}\right)$ such that $\eta \geq \mathbf{1}_{E}$, we have

$$
\begin{aligned}
\mu(E) & \leq \int_{Q_{T}} \eta d \mu=\Phi(\eta)=\int_{Q_{T}} \eta f^{0} \rho^{2}-\int_{Q_{T}} \bar{f} \nabla\left(\rho^{2} \eta\right) \\
& \leq C\left(\|\nabla \eta\|_{2, \rho, T}+\|\eta\|_{2, \rho, T}^{2}\right) .
\end{aligned}
$$

Thus, $\mu \ll \overline{\operatorname{cap}}_{\check{Q}_{T}}$. Now, for $E \subset \subset \mathbb{R}^{d}$ define

$$
\operatorname{cap}_{\mathbb{R}^{d}}(E)=\inf \left\{\int_{\mathbb{R}^{d}}|\nabla \eta(x)|^{2} d x+\int_{\mathbb{R}^{d}}|\eta(x)|^{2} d x: \eta \in C_{0}^{\infty}\left(\mathbb{R}^{d}\right), \eta \geq \mathbf{1}_{E}\right\},
$$

and extend it in the standard way to $\mathcal{B}\left(\mathbb{R}^{d}\right)$. From [5] it follows that for every $B \in$ $\mathcal{B}\left(\check{Q}_{T}\right)$,

$$
\overline{\operatorname{cap}}_{\check{Q}_{T}}(B)=\int_{0}^{T} \operatorname{cap}_{\mathbb{R}^{d}}\left(B_{t}\right) d t,
$$

where $B_{t}=\left\{x \in \mathbb{R}^{d} ;(t, x) \in B\right\}$. Since $\mu \ll \overline{\text { cap }}_{\check{Q}_{T}}$, using the well known fact that elements of $H_{\rho}^{1}$ have quasi-continuous versions defined up to the sets of cap $_{\mathbb{R}^{d}}$-measure zero (see [18, Chap. 2]), we may extend formula (2.1) to all $\eta \in$ $\mathbb{L}_{2}\left(0, T ; H_{\rho}^{1}\right)$.

It is worth noting that in the definition of capacity $\overline{c a p}_{Q_{T}}$ and in the representation theorem for functionals in $\mathbb{L}_{2}\left(0, T ; H_{\rho}^{-1}\right)$ derivatives with respect to the time variable do not appear. Therefore, various facts on functionals $\mu \in \mathbb{L}_{2}\left(0, T ; H_{\rho}^{-1}\right) \cap \mathcal{M}$ can be proven by making obvious changes in proofs of corresponding facts concerning elliptic capacity and functionals in $H_{\rho}^{-1}$. In particular, slightly modifying arguments from [6] and [10], one can prove the following theorems.

Theorem 2.1 Let $\mu \in \mathcal{M}$. If $\mu \ll \overline{\operatorname{cap}}_{Q_{T}}$ then there exist $\gamma_{1}, \gamma_{2} \in \mathbb{L}_{2}\left(0, T ; H_{\rho}^{-1}\right) \cap$ $\mathcal{M}^{+}$and positive $\alpha_{i} \in \mathbb{L}_{1, \operatorname{loc}}\left(Q_{T}, \gamma_{i}\right), i=1,2$ such that $d \mu=\alpha_{1} d \gamma_{1}-\alpha_{2} d \gamma_{2}$.

Theorem 2.2 A Radon measure $\mu$ vanishes on sets of zero $\overline{\operatorname{cap}}_{Q_{T}}$ capacity if and only if it admits the decomposition

$$
\mu=\Phi+k,
$$

where $\Phi \in \mathbb{L}_{2}\left(0, T ; H_{\rho}^{-1}\right)$ and $k \in \mathbb{L}_{1}^{\text {loc }}\left(Q_{T}\right)$. 
In the paper, we will also use another notion of capacity, the so-called parabolic capacity, which appears when considering the natural space of strong solutions of variational inequalities, i.e., the space $\mathcal{W}_{\rho}$.

Let $\Omega=C\left([0, T], \mathbb{R}^{d}\right)$ denote the space of continuous $\mathbb{R}^{d}$-valued functions on $[0, T]$ equipped with the topology of uniform convergence and let $X$ be the canonical process on $\Omega$. It is known that for a given operator $L_{t}$ defined by (1.1) with $a$ and $b$ satisfying (1.2) one can construct a weak fundamental solution $p$ for $L_{t}$ and then a Markov family $\mathbb{X}=\left\{\left(X, P_{s, x}\right) ;(s, x) \in Q_{\hat{T}}\right\}$ for which $p$ is the transition density function, i.e.,

$$
P_{s, x}\left(X_{t}=x ; 0 \leq t \leq s\right)=1, \quad P_{s, x}\left(X_{t} \in \Gamma\right)=\int_{\Gamma} p(s, x, t, y) d y, \quad t \in(s, T]
$$

for any $\Gamma \in \mathcal{B}\left(\mathbb{R}^{d}\right)$ (see $\left.[29,37]\right)$. We define the parabolic capacity of an open set $B \subset Q_{\hat{T}}$ by

$$
\operatorname{cap}_{L}(B)=\int_{0}^{T} P_{s, m}\left(\exists t \in(s, T):\left(t, X_{t}\right) \in B\right) d s,
$$

where $m$ is the Lebesgue measure on $\mathbb{R}^{d}$ and

$$
P_{s, m}(\Gamma)=\int_{\mathbb{R}^{d}} P_{s, x}(\Gamma) d x, \quad \Gamma \in \mathcal{G} .
$$

It is known (see [18, Theorem A.1.2, Lemma A.2.5, A.2.6]) that such a defined set function might be uniquely extended to a Choquet capacity on $\mathcal{B}\left(Q_{\hat{T}}\right)$ and it satisfies (2.3) for every compact set $K \subset Q_{\hat{T}}$. In what follows, we say that some property is satisfied quasi-everywhere (q.e. for short) if it is satisfied except of a Borel set of zero capacity $\operatorname{cap}_{L}$.

Remark 2.3 It follows directly from the definition of $\operatorname{cap}_{L}$ that $\operatorname{cap}_{L}(\{s\} \times B)>0$ for every $s \in(0, T)$ and $B \in \mathcal{B}\left(\mathbb{R}^{d}\right)$ such that $m(B)>0$. Hence, if some property holds for q.e. $(s, x) \in Q_{\hat{T}}$, then it holds for a.e. $x \in \mathbb{R}^{d}$ for every $s \in(0, T)$.

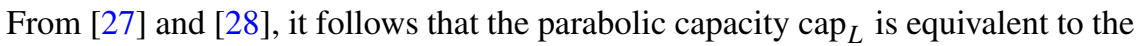
following parabolic capacity cap 2 in the analytical sense.

Definition 2.4 Let $V \subset Q_{\hat{T}}$ be an open set. We set

$$
\operatorname{cap}_{2}(V)=\inf \left\{\|u\|_{\mathcal{W}_{\rho}}: u \in \mathcal{W}_{\rho}, u \geq \mathbf{1}_{V} \text { a.e. }\right\}
$$

with the convention that $\inf \emptyset=\infty$. The parabolic capacity of a Borel set $B \subset Q_{\hat{T}}$ is defined by

$$
\operatorname{cap}_{2}(B)=\inf \left\{\operatorname{cap}_{2}(V): V \text { is an open subset of } Q_{T}, B \subset V\right\} .
$$

From [28, Proposition 2], it follows that cap $_{2}$ is a Choquet capacity. 
Remark 2.5 If cap $_{L}$ is a Choquet capacity, it follows in particular (see [18, Theorem A.1.1.]) that for any $B \in \mathcal{B}\left(Q_{\hat{T}}\right)$,

$$
\operatorname{cap}_{L}(B)=\sup _{K \subset B, K \text {-compact }} \operatorname{cap}_{L}(K)
$$

which implies that $\operatorname{cap}_{L}(B)=0$ iff $\operatorname{cap}_{L}(K)=0$ for every compact subset $K$ of $B$.

Definition 2.6 We say that $u: Q_{T} \rightarrow \mathbb{R}$ is quasi-continuous if $u$ is Borel measurable and $[0, T] \ni t \mapsto u\left(t, X_{t}\right)$ is a continuous process under the measure $P_{s, x}$ for q.e. $(s, x) \in Q_{\hat{T}}$.

The notion of quasi-continuity defined above is equivalent to the following one: for every $\varepsilon>0$ there exists an open set $U_{\varepsilon} \subset \check{Q}_{T}$ such that $u_{\mid \check{Q}_{T} \backslash U_{\varepsilon}}$ is continuous and $\operatorname{cap}_{2}\left(U_{\varepsilon}\right)<\varepsilon$ (see Remark 3.6 and Proposition 3.7 in [35]). Let us also note that it is known that every $u \in \mathcal{W}_{\rho}$ has a quasi-continuous version (see [27]).

\section{Diffusions Corresponding to Divergence Form Operators}

Set $\mathcal{F}_{t}^{s}=\sigma\left(X_{u}, u \in[s, t]\right), \overline{\mathcal{F}}_{t}^{s}=\sigma\left(X_{u}, u \in[T+s-t, T]\right)$ and define $\mathcal{G}$ as the completion of $\mathcal{F}_{T}^{S}$ with respect to the family $\mathcal{P}=\left\{P_{s, \mu}: \mu\right.$ is a probability measure on $\left.\mathcal{B}\left(\mathbb{R}^{d}\right)\right\}$, where $P_{s, \mu}(\cdot)=\int_{\mathbb{R}^{d}} P_{s, x}(\cdot) \mu(d x)$, and define $\mathcal{G}_{t}^{s}\left(\overline{\mathcal{G}}_{t}^{s}\right)$ as the completion of $\mathcal{F}_{t}^{s}\left(\overline{\mathcal{F}}_{t}^{s}\right)$ in $\mathcal{G}$ with respect to $\mathcal{P}$.

We will say that a family $A=\left\{A_{s, t}, 0 \leq s \leq t \leq T\right\}$ of random variables is an additive functional (AF) of $\mathbb{X}$ if $A_{s,}$. is a $\left(\left\{\mathcal{G}_{t}^{s}\right\}, P_{s, x}\right)$-measurable càdlàg process and $P_{s, x}\left(A_{s, t}=A_{s, u}+A_{u, t}, s \leq u \leq t \leq T\right)=1$ for q.e. $(s, x) \in Q_{\hat{T}}$. If, in addition, $A_{s}$, has $P_{s, x}$-almost all continuous trajectories for q.e. $(s, x) \in Q_{\hat{T}}$, then $A$ is called a continuous $\mathrm{AF}(\mathrm{CAF})$, and if $A_{s,}$ is an increasing process under $P_{s, x}$ for q.e. $(s, x) \in Q_{\hat{T}}$, it is called an increasing $\mathrm{AF}$ or positive AF. If $M$ is an AF such that for q.e. $(s, x) \in Q_{\hat{T}}, E_{s, x}\left|M_{s, t}\right|^{2}<\infty$ and $E_{s, x} M_{s, t}=0$ for $t \in[s, T]$ ( $E_{s, x}$ is the expectation with respect to $P_{s, x}$ ), it is called a martingale AF (MAF). We say that $A$ is an $\mathrm{AF}(\mathrm{CAF}$, increasing $\mathrm{AF}, \mathrm{MAF})$ in the strict sense if the corresponding property holds for every $(s, x) \in Q_{\hat{T}}$. Finally, we say that $A$ is a quasi-strict AF (CAF, increasing AF, MAF) if the corresponding property holds under $P_{s, x}$ for every $(s, x) \in Q_{\hat{T}}$ on $(s, T]$ and for q.e. $(s, x) \in Q_{\hat{T}}$ on $[s, T]$. Since in what follows, except for Proposition 3.3, we will consider exclusively quasi-strict AFs, we will call it briefly additive functionals.

\subsection{Fukushima's Decomposition and Decomposition in the Sense of Föllmer}

It is known (see $[22,32])$ that there exist a CAF $A$ in the strict sense and a continuous MAF $M$ in the strict sense such that

$$
X_{t}-X_{s}=M_{s, t}+A_{s, t}, \quad t \in[s, T], P_{s, x} \text {-a.s. }
$$




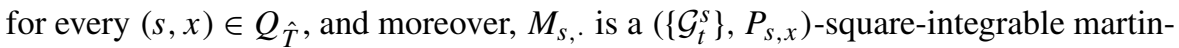
gale on $[s, T]$ with the covariation given by

$$
\left\langle M_{s, \cdot}^{i}, M_{s, \cdot}^{j}\right\rangle_{t}=\int_{s}^{t} a_{i j}\left(\theta, X_{\theta}\right) d \theta, \quad t \in[s, T], i, j=1, \ldots, d,
$$

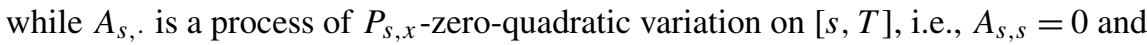

$$
\sum_{t_{i} \in \Pi_{m}}\left|A_{s, t_{i+1}}-A_{s, t_{i}}\right|^{2} \rightarrow 0 \quad \text { in probability } P_{s, x}
$$

for any sequence $\left\{\Pi_{m}=\left\{t_{0}, t_{1}, \ldots, t_{i(m)}\right\}\right\}$ of partitions of $[s, T]$ such that $s=t_{0}<$ $t_{1}<\cdots<t_{i(m)}=T$ and $\left\|\Pi_{m}\right\|=\max _{1 \leq i \leq i(m)}\left|t_{i}-t_{i-1}\right| \rightarrow 0$ as $m \rightarrow \infty$. In particular, $X .-X_{s}$ is a $\left(\left\{\mathcal{G}_{t}^{s}\right\}, P_{s, x}\right)$-Dirichlet process in the sense of Föllmer. One can also show that $M$ is an MAF of locally zero-energy and $A$ is a CAF of locally finite energy (see [32] and [30, 31] for time-homogeneous diffusions), i.e., (3.1) coincides with Fukushima's decomposition for $\mathbb{X}$.

Observe that if $\sigma \sigma^{*}=a$ then, by (3.2),

$$
B_{s, t}=\int_{s}^{t} \sigma^{-1}\left(\theta, X_{\theta}\right) d M_{s, \theta}, \quad t \in[s, T]
$$

is a $\left(\left\{\mathcal{G}_{t}^{s}\right\}, P_{s, x}\right)$-Wiener process.

\subsection{Lyons-Zheng's Decomposition}

Additional information on the structure of $A$ of decomposition (3.1) provides the Lyons-Zheng's decomposition for $\mathbb{X}$. Let $(s, x) \in Q_{\hat{T}}$. For $s \leq u \leq t \leq T$ we set

$$
\alpha_{u, t}^{s, x, i}=\sum_{j=1}^{d} \int_{u}^{t} \frac{1}{2} a_{i j}\left(\theta, X_{\theta}\right) p^{-1} \frac{\partial p}{\partial y_{j}}\left(s, x, \theta, X_{\theta}\right) d \theta, \quad \beta_{u, t}^{i}=\int_{u}^{t} b^{i}\left(\theta, X_{\theta}\right) d \theta
$$

In the sequel, for a process $Y$ on $[s, T]$ and fixed measure $P_{s, x}$ we write $\bar{Y}_{t}=$ $Y_{T+s-t}$ for $t \in[s, T]$.

From [32] it follows that under $P_{s, x}$ the canonical process $X$ admits the decomposition

$$
X_{t}-X_{u}=\frac{1}{2} M_{u, t}+\frac{1}{2}\left(N_{s, T+s-t}^{s, x}-N_{s, T+s-u}^{s, x}\right)-\alpha_{u, t}^{s, x}+\beta_{u, t}, \quad s \leq u \leq t \leq T,
$$

where $M_{s, .}$ is the martingale of (3.1) and $N_{s, \cdot}^{s, x}$ is a $\left(\left\{\overline{\mathcal{G}}_{t}^{s}\right\}, P_{s, x}\right)$-martingale such that

$$
\left\langle N_{s, \cdot}^{s, x, i}, N_{s, \cdot}^{s, x, j}\right\rangle_{t}=\int_{s}^{t} a_{i j}\left(\bar{\theta}, \bar{X}_{\theta}\right) d \theta, \quad t \in[s, T], i, j=1, \ldots, d .
$$

Observe that the covariation of $N^{s, x}$ does not depend on $x \in \mathbb{R}^{d}$. 
Remark 3.1 From (2.7) in [29] it follows that

$$
E_{s, x}\left(\tilde{N}_{u_{1}, u_{2}}^{s, x} \mid \overline{\mathcal{G}}_{T+s-u_{2}}^{s}\right)=0, \quad s \leq u_{1} \leq u_{2} \leq T
$$

where $\tilde{N}_{u, t}^{s, x}=N_{s, T+s-t}^{s, x}-N_{s, T+s-u}^{s, x}$. Hence, if we put

$$
\bar{M}_{u, t}^{s, x}=-\left(N_{s, T+s-t}^{s, x}-N_{s, T+s-u}^{s, x}\right),
$$

then for every $t \in[s, T),\left\{\bar{M}_{t+s-u, t}, u \in[s, t]\right\}$ is a $\left(\left\{\overline{\mathcal{G}}_{T}^{t+s-u}\right\}_{u \in[s, t]}, P_{s, x}\right)$-martingale and under $P_{S, x}$ the process $X$ admits the decomposition

$$
X_{t}-X_{u}=\frac{1}{2} M_{u, t}-\frac{1}{2} \bar{M}_{u, t}^{s, x}-\alpha_{u, t}^{s, x}+\beta_{u, t}, \quad s \leq u \leq t \leq T
$$

considered in [24].

\subsection{Forward-Backward Integrals}

Let $\bar{f}=\left(f_{1}, \ldots, f_{d}\right): Q_{T} \rightarrow \mathbb{R}^{d}$ and let $S$ be some class of real functions defined on $Q_{T}$. To simplify notation, in what follows we write $\bar{f} \in S$ if $f_{i} \in S, i=1, \ldots, d$.

Let $\bar{f} \in \mathcal{B}_{b}^{\text {loc }}\left(Q_{T}\right)$. Similarly to $[34,36]$, using (3.5) we set under the measure $P_{s, x}$,

$$
\int_{r}^{t} \bar{f}\left(\theta, X_{\theta}\right) d^{*} X_{\theta} \equiv-\int_{r}^{t} \bar{f}\left(\theta, X_{\theta}\right)\left(d M_{s, \theta}+d \alpha_{s, \theta}^{s, x}\right)-\int_{T+s-t}^{T+s-r} \bar{f}\left(\bar{\theta}, \bar{X}_{\theta}\right) d N_{s, \theta}^{s, x}
$$

for $s \leq u \leq t \leq T$, where $\bar{\theta}=T+s-\theta$. By Proposition 7.6, all integrals on the right-hand side of (3.7) are well defined for every $(s, x) \in Q_{\hat{T}}$. The interest in the integral defined above comes from the fact that if $\bar{f}$ is regular then

$$
\int_{u}^{t} \operatorname{div} \bar{f}\left(\theta, X_{\theta}\right) d \theta=\int_{u}^{t} a^{-1} \bar{f}\left(\theta, X_{\theta}\right) d^{*} X_{\theta}, \quad s \leq u \leq t \leq T, P_{s, x} \text {-a.s. }
$$

(see [34]), which enables one to extend the integral on the left-hand side of (3.8) to $\bar{f} \in \mathcal{B}_{b}^{\text {loc }}\left(Q_{T}\right)$.

Our first goal is to extend the class of functions for which (3.7) is well defined for q.e. $(s, x) \in Q_{\hat{T}}$. In view of (1.2), (3.2), (3.6), Proposition 7.6 and Corollary 3.4, to define integrals with respect to the forward and backward martingales it suffices to assume that $f \in \mathbb{L}_{2, \rho}\left(Q_{T}\right)$. The main problem is to define the integral with respect to $\alpha^{s, x}$ because the gradient of $p$ is not square-integrable (see [1]) and $\alpha^{s, x}$ depends on $(s, x)$. The latter fact makes difficulties in applying the Markov property of $\mathbb{X}$ to get the existence of the integral.

We start with the investigation of integrals with respect to $\alpha^{s, x}$ in case of timehomogeneous diffusions. Let $\left\{\left(X, P_{x}\right) ; x \in \mathbb{R}^{d}\right\}$ be a Hunt process associated with the Dirichlet form (1.5). 
It is known that if $a$ is piecewise smooth (see [11] for details) then there exists an $M>0$ such that

$$
\left|\nabla_{x} p(t, x, y)\right| \leq \frac{M}{t^{(d+1) / 2}} \exp \left(-\frac{|y-x|^{2}}{2 M t}\right) .
$$

Hence, by the elementary calculations,

$$
E_{x} \int_{0}^{T}\left|f\left(X_{t}\right)\right| d\left|\alpha^{x}\right|_{t} \leq C \int_{\mathbb{R}^{d}} f(y)|y-x|^{1-d} d y .
$$

On the right-hand side of the above inequality, we recognize the Riesz potential of order 1. Therefore, repeating the arguments from the proof of [15, Proposition 3.6] shows that for every $f \in \mathbb{L}_{2, \text { loc }}\left(\mathbb{R}^{d}\right)$,

$$
P_{x}\left(\int_{0}^{T}\left|f\left(X_{t}\right)\right| d\left|\alpha^{x}\right|_{t}<\infty\right)=1
$$

for $\mathcal{E}$-q.e. $x \in \mathbb{R}^{d}$.

The following example shows that in the time-dependent case the condition $f \in$ $\mathbb{L}_{2, \rho}\left(Q_{T}\right)$ is insufficient to guarantee (3.9) even if $a$ is smooth.

Example 3.2 Let $d=1, a=1, b=0$, so that $X_{t}, t>s$, has under $P_{s, x}$ the normal distribution with mean $x$ and variance $t-s$. Then

$$
\alpha_{s, t}^{s, x}=\frac{1}{2} \int_{s}^{t} p^{-1} \frac{\partial p}{\partial y}\left(s, x, \theta, X_{\theta}\right) d \theta=\frac{1}{2} \int_{s}^{t} \frac{x-X_{\theta}}{\theta-s} d \theta .
$$

Suppose that $f$ is nonnegative and does not depend on $x$. Then

$$
\begin{aligned}
w(s, x) & =E_{s, x} \int_{s}^{T} f(\theta) d\left|\alpha_{s, \cdot}^{s, x}\right|_{\theta}=E_{s, x} \int_{s}^{T} f(\theta) \frac{\left|x-X_{\theta}\right|}{\theta-s} d \theta \\
& =C \int_{s}^{T} \frac{f(\theta)}{(\theta-s)^{1 / 2}} d \theta,
\end{aligned}
$$

i.e., $w(s, x)$ does not depend on $x$. Now, let us fix $t_{0} \in(0, T)$. Since the function $\left(t_{0}, T\right) \ni t \mapsto\left(t-t_{0}\right)^{-1 / 2}$ does not belong to $\mathbb{L}_{2}\left(t_{0}, T\right)$, one can find $f \in \mathbb{L}_{2}(0, T)$ such that $\int_{t_{0}}^{T} f(\theta)\left(\theta-t_{0}\right)^{-1 / 2}=\infty$. Then $w=\infty$ on the set $\left\{t_{0}\right\} \times \mathbb{R}^{d}$ and from Remark 2.3 it follows that $\operatorname{cap}_{L}\left(\left\{t_{0}\right\} \times \mathbb{R}^{d}\right)>0$.

We will extend the integral side of (3.7) to $\bar{f} \in \mathbb{L}_{2, \rho}\left(Q_{T}\right)$ by using approximation.

Proposition 3.3 Let $p>0$ and let $A, A^{n}, n \in \mathbb{N}$, be CAFs of $\mathbb{X}$ such that

$$
E_{s, x} \sup _{s \leq t \leq T}\left|A_{s, t}^{n}-A_{s, t}\right|^{p} \rightarrow 0
$$

for a.e. $(s, x) \in Q_{\hat{T}}$. Then there exists a subsequence $\left\{n^{\prime}\right\}$ of $\{n\}$ such that (3.10) holds along $\left\{n^{\prime}\right\}$ for q.e. $(s, x) \in Q_{\hat{T}}$. 
Proof Set $B=\left\{(s, x): E_{s, x} \sup _{s \leq t \leq T}\left|A_{s, t}^{n}-A_{s, t}\right|^{p} \nrightarrow 0\right\}$ and let $\tau=\inf \{t \in[s, T)$ : $\left.\left(t, X_{t}\right) \in K\right\}$, where $K$ is a compact subset of $B$. Since $\left(X, P_{s, x}\right)$ is a Feller process, $\tau$ is a $\left\{\mathcal{G}_{t}^{s}\right\}$-stopping time. Hence, by the strong Markov property with random shift and additivity of $A^{n}$ and $A$,

$$
\begin{aligned}
P_{s, x}(\tau<\infty) & =P_{s, x}\left(E_{\tau, X_{\tau}} \sup _{\tau \leq t \leq T}\left|A_{\tau, t}^{n}-A_{\tau, t}\right|^{p} \nrightarrow 0, \tau<\infty\right) \\
& =P_{s, x}\left(E_{s, x}\left(\sup _{\tau \leq t \leq T}\left|A_{\tau, t}^{n}-A_{\tau, t}\right|^{p} \mid \mathcal{G}_{\tau}^{s}\right) \nrightarrow 0, \tau<\infty\right) \\
& \leq P_{s, x}\left(2^{p \wedge 1} E_{s, x}\left(\sup _{s \leq t \leq T}\left|A_{s, t}^{n}-A_{s, t}\right|^{p} \mid \mathcal{G}_{\tau \wedge T}^{s}\right) \nrightarrow 0\right) .
\end{aligned}
$$

Set

$$
T_{n, m}(s, x, \omega)=E_{s, x}\left(\sup _{s \leq t \leq T}\left|A_{s, t}^{n}-A_{s, t}\right|^{p} \mid \mathcal{G}_{\tau \wedge T}^{s}\right)(\omega) .
$$

By (3.10), $T_{n, m} \rightarrow 0$ in $\mathbb{L}_{1}\left(Q_{T} \times \Omega, \Pi\right)$, where $\Pi$ is the finite measure defined by the formula

$$
\Pi(B)=\int_{Q_{T}}\left(E_{s, x} \mathbf{1}_{B}(s, x)\right) \rho(x) d s d x .
$$

Using the Borel-Cantelli lemma, we can choose a subsequence (still denoted by $\{n\})$ such that $T_{n, m} \rightarrow 0, \Pi$-a.e. In particular, $T_{n, m}(s, x) \rightarrow 0, P_{s, x}$-a.s. for a.e. $(s, x) \in Q_{T}$. Hence $P_{s, x}(\tau<\infty)=0$ for a.e. $(s, x) \in Q_{T}$, and consequently $\operatorname{cap}_{L}(K)=0$. Hence, by the fact that cap ${ }_{L}$ is a Choquet capacity, $\operatorname{cap}_{L}(B)=0$.

Corollary 3.4 Let $p>0$ and let $A$ be a CAF of $\mathbb{X}$ such that

$$
E_{s, x} \sup _{s \leq t \leq T}\left|A_{s, t}\right|^{p}<\infty
$$

for a.e. $(s, x) \in Q_{\hat{T}}$. Then (3.11) holds for q.e. $(s, x) \in Q_{\hat{T}}$.

Proposition 3.5 Let $\bar{f} \in \mathbb{L}_{2, \rho}\left(Q_{T}\right)$. Then there exists a unique CAF $D$ of $\mathbb{X}$ such that for every sequence $\left\{\bar{f}_{n}\right\} \subset \mathcal{B}_{b}\left(Q_{T}\right)$ convergent to $\bar{f}$ in $\mathbb{L}_{2, \rho}\left(Q_{T}\right)$ there exists a subsequence (still denoted by $\{n\})$ such that

$$
E_{s, x} \sup _{s \leq t \leq T}\left|\int_{s}^{t} \bar{f}_{n}\left(\theta, X_{\theta}\right) d^{*} X_{\theta}-D_{s, t}\right| \rightarrow 0
$$

for q.e. $(s, x) \in Q_{\hat{T}}$.

Proof Put $A_{s, t}^{n}=\int_{s}^{t} \bar{f}_{n}\left(\theta, X_{\theta}\right) d^{*} X_{\theta}$. By Proposition 7.6,

$$
\int_{Q_{T}}\left(E_{s, x} \sup _{s \leq t \leq T}\left|A_{s, t}^{n}-A_{s, t}^{m}\right|\right) \rho(x) d x \rightarrow 0 .
$$


Hence, by Proposition 3.3, there exists a subsequence (still denoted by $\{n\}$ ) and some process $D^{s, x}$ such that $E_{s, x} \sup _{s \leq t \leq T}\left|A_{s, t}^{n}-D_{s, t}^{s, x}\right| \rightarrow 0$ for q.e. $(s, x) \in Q_{\hat{T}}$. Using arguments from the proof of [18, Lemma A.3.2], one can choose a version of $D^{s, x}$ which does not depend on $(s, x)$.

To prove uniqueness, suppose that $\tilde{D}$ is another process having the properties of $D$. Let $\bar{g}_{n}, \bar{f}_{n} \in \mathcal{B}_{b}\left(Q_{T}\right), \bar{f}_{n}, \bar{g}_{n} \rightarrow \bar{f}$ in $\mathbb{L}_{2, \rho}\left(Q_{T}\right)$. Let $\{n\}$ be a subsequence such that (3.12) holds with the pairs $\left(\bar{f}_{n}, D\right),\left(\bar{g}_{n}, \tilde{D}\right)$ and $\left(\bar{f}_{n}, \bar{g}_{n}\right)$. For the latter pair, it is possible thanks to Proposition 3.3 and the following convergence

$$
\int_{Q_{T}}\left(E_{s, x} \sup _{s \leq t \leq T}\left|\int_{s}^{t} \bar{f}_{n}\left(\theta, X_{\theta}\right) d^{*} X_{\theta}-\int_{s}^{t} \bar{g}_{n}\left(\theta, X_{\theta}\right) d^{*} X_{\theta}\right|\right) \rho(x) d x \rightarrow 0,
$$

which is a consequence of convergence of $\left\{\bar{f}_{n}\right\},\left\{\bar{g}_{n}\right\}$ and Proposition 7.6. Finally, for q.e. $(s, x) \in Q_{\hat{T}}$,

$$
\begin{aligned}
& E_{s, x} \sup _{s \leq t \leq T}\left|D_{s, t}-\tilde{D}_{s, t}\right| \\
& \leq \lim _{n \rightarrow \infty} E_{s, x} \sup _{s \leq t \leq T}\left|\int_{s}^{t} \bar{f}_{n}\left(\theta, X_{\theta}\right) d^{*} X_{\theta}-D_{s, t}\right| \\
& \quad+\lim _{n \rightarrow \infty} E_{s, x} \sup _{s \leq t \leq T}\left|\int_{s}^{t} \bar{f}_{n}\left(\theta, X_{\theta}\right) d^{*} X_{\theta}-\int_{s}^{t} \bar{g}_{n}\left(\theta, X_{\theta}\right) d^{*} X_{\theta}\right| \\
& \quad+\lim _{n \rightarrow \infty} E_{s, x} \sup _{s \leq t \leq T}\left|\int_{s}^{t} \bar{g}_{n}\left(\theta, X_{\theta}\right) d^{*} X_{\theta}-\tilde{D}_{s, t}\right|=0,
\end{aligned}
$$

and the proof is complete.

Remark 3.6 For every $(s, x) \in Q_{\hat{T}}$ and $s<r \leq t \leq T$, the integrals on the right-hand side of (3.7) are well defined $P_{s, x}$-a.s. This follows from Aronson's estimates and Proposition 7.4(ii) because

$$
\begin{aligned}
E_{s, x} \int_{r}^{T}\left|\bar{f}\left(\theta, X_{\theta}\right)\right|^{2} d \theta & =\int_{Q_{r T}}|\bar{f}(\theta, y)|^{2} p(s, x, \theta, y) d \theta d y \\
& \leq C \int_{Q_{r T}} \frac{1}{(\theta-s)^{d / 2}}|\bar{f}(\theta, y)|^{2} \exp \left(\frac{-|y-x|}{C(\theta-s)}\right) \\
& \leq C \frac{\rho^{-1}(x)}{(r-s)^{d / 2}}\|\bar{f}\|_{2, \rho, T}^{2}
\end{aligned}
$$

and

$$
\begin{aligned}
E_{s, x} \int_{r}^{t}\left|\bar{f}\left(\theta, X_{\theta}\right)\right| d\left|\alpha_{s, \cdot}^{s, x}\right|_{\theta} & \leq \int_{Q_{r, T}}\left|\bar{f}(\theta, y) \| \nabla_{x} p\right|(s, x, \theta, y) d \theta d y \\
& \leq\|\bar{f}\|_{2, \rho, T}\|\nabla p(s, x)\|_{2, \rho^{-1}, r, T}
\end{aligned}
$$


Proposition 3.7 Let $\bar{f} \in \mathbb{L}_{2, \rho}\left(Q_{T}\right)$ and let $D$ be the CAF of Proposition 3.5. Then $P_{s, x}$-a.s.,

$D_{r, t}=-\int_{r}^{t} \bar{f}\left(\theta, X_{\theta}\right)\left(d M_{s, \theta}+d \alpha_{s, \theta}^{s, x}\right)-\int_{T+s-t}^{T+s-r} \bar{f}\left(\bar{\theta}, \bar{X}_{\theta}\right) d N_{s, \theta}^{s, x}, \quad s<r \leq t \leq T$

for q.e. $(s, x) \in Q_{\hat{T}}$.

Proof Let $\left\{\bar{f}_{n}\right\} \subset \mathcal{B}_{b}\left(Q_{T}\right)$ be such that (3.12) holds q.e. Then by (3.7),

$\int_{r}^{t} \bar{f}_{n}\left(\theta, X_{\theta}\right) d^{*} X_{\theta}=-\int_{r}^{t} \bar{f}_{n}\left(\theta, X_{\theta}\right)\left(d M_{s, \theta}+d \alpha_{s, \theta}^{s, x}\right)-\int_{T+s-t}^{T+s-r} \bar{f}_{n}\left(\bar{\theta}, \bar{X}_{\theta}\right) d N_{s, \theta}^{s, x}$,

and the result follows from (3.12), (3.14), and (3.15).

Put $N=N_{1} \cup N_{2}$, where

$$
\begin{aligned}
& N_{1}=\left\{(s, x) \in Q_{\hat{T}} ; P_{s, x}\left(\int_{s}^{T}\left|\bar{f}\left(t, X_{t}\right)\right|^{2} d t<\infty\right)=1\right\}^{c}, \\
& N_{2}=\left\{(s, x) \in Q_{\hat{T}} ; \text { p.v. }-\int_{s}^{T} \bar{f}\left(t, X_{t}\right) d \alpha_{s, t}^{s, x} \text { exists and is finite } P_{s, x} \text {-a.s. }\right\}^{c}
\end{aligned}
$$

and

$$
\text { p.v.- } \int_{s}^{T} \bar{f}\left(t, X_{t}\right) d \alpha_{s, t}^{s, x} \equiv \lim _{\delta \rightarrow 0^{+}} \int_{s+\delta}^{T} \bar{f}\left(t, X_{t}\right) d \alpha_{s, t}^{s, x} \text {. }
$$

Corollary 3.8 If $f \in \mathbb{L}_{2, \rho}\left(Q_{\hat{T}}\right)$ then $\operatorname{cap}_{L}(N)=0$.

Proof Follows directly from Proposition 3.5 and Proposition 3.7.

Let $\bar{f} \in \mathbb{L}_{2, \rho}\left(Q_{T}\right)$. For a fixed $(s, x) \in Q_{\hat{T}}$, we set

$$
\int_{r}^{t} \bar{f}\left(\theta, X_{\theta}\right) d^{*} X_{\theta}=-\int_{r}^{t} \bar{f}\left(\theta, X_{\theta}\right)\left(d M_{s, \theta}+d \alpha_{s, \theta}^{s, x}\right)-\int_{T+s-t}^{T+s-r} \bar{f}\left(\bar{\theta}, \bar{X}_{\theta}\right) d N_{s, \theta}^{s, x}
$$

for all $0 \leq s<r \leq t \leq T$, and for a fixed $(s, x) \in N^{c}$ we set

$$
\begin{aligned}
\int_{s}^{t} \bar{f}\left(\theta, X_{\theta}\right) d^{*} X_{\theta}= & -\int_{s}^{t} \bar{f}\left(\theta, X_{\theta}\right) d M_{s, \theta} \\
& + \text { p.v.- } \int_{s}^{t} \bar{f}\left(\theta, X_{\theta}\right) d \alpha_{s, \theta}^{s, x}-\int_{T+s-t}^{T} \bar{f}\left(\bar{\theta}, \bar{X}_{\theta}\right) d N_{s, \theta}^{s, x}
\end{aligned}
$$

for all $0 \leq s \leq t \leq T$.

Under stronger integrability conditions on $f$, all integrals on the right-hand side of (3.7) are defined for q.e. $(s, x)$. 
Proposition 3.9 If $f \in \mathbb{L}_{p, \rho}\left(Q_{T}\right)$ for some $p>2$ then for q.e. $(s, x) \in Q_{\hat{T}}$,

$$
\begin{aligned}
\int_{s}^{t} \bar{f}\left(\theta, X_{\theta}\right) d^{*} X_{\theta}= & -\int_{s}^{t} \bar{f}\left(\theta, X_{\theta}\right)\left(d M_{s, \theta}+d \alpha_{s, \theta}^{s, x}\right) \\
& -\int_{T+s-t}^{T} \bar{f}\left(\bar{\theta}, \bar{X}_{\theta}\right) d N_{s, \theta}^{s, x}, \quad t \in[s, T], P_{s, x} \text {-a.s. }
\end{aligned}
$$

Proof By (7.3),

$$
E_{s, x} \int_{s}^{T}\left|\bar{f}\left(t, X_{t}\right)\right| d\left|\alpha_{s, \cdot}^{s, x}\right|_{t} \leq C(p)\left(E_{s, x} \int_{s}^{T}\left|\bar{f}\left(t, X_{t}\right)\right|^{p} d t\right)^{2 / p} .
$$

From Proposition 7.6, it follows that the right-hand side is finite for a.e. $(s, x) \in Q_{\hat{T}}$. Hence, by Corollary 3.4, it is finite for q.e. $(s, x) \in Q_{\hat{T}}$. The result now follows from Corollary 3.8.

Proposition 3.10 Let $\left\{\bar{f}_{n}\right\} \subset \mathbb{L}_{2, \rho}\left(Q_{T}\right)$ and $\bar{f}_{n} \rightarrow \bar{f}$ in $\mathbb{L}_{2, \rho}\left(Q_{T}\right)$. Then

(i) For every $(s, x) \in Q_{\hat{T}}$ and $r \in(s, T]$,

$$
E_{S, x} \sup _{r \leq t \leq T}\left|\int_{r}^{t} \bar{f}_{n}\left(\theta, X_{\theta}\right) d^{*} X_{\theta}-\int_{r}^{t} \bar{f}\left(\theta, X_{\theta}\right) d^{*} X_{\theta}\right| \rightarrow 0
$$

(ii) There exists a subsequence (still denoted by $\{n\})$ such that for q.e. $(s, x) \in Q_{\hat{T}}$,

$$
E_{s, x} \sup _{s \leq t \leq T}\left|\int_{s}^{t} \bar{f}_{n}\left(\theta, X_{\theta}\right) d^{*} X_{\theta}-\int_{s}^{t} \bar{f}\left(\theta, X_{\theta}\right) d^{*} X_{\theta}\right| \rightarrow 0 .
$$

Proof (i) follows easily from (3.14) and (3.15). (ii) follows from Proposition 3.3 because

$$
\begin{aligned}
& \int_{Q_{T}}\left(E_{s, x} \sup _{s \leq t \leq T}\left|\int_{s}^{t} \bar{f}_{n}\left(\theta, X_{\theta}\right) d^{*} X_{\theta}-\int_{s}^{t} \bar{f}\left(\theta, X_{\theta}\right) d^{*} X_{\theta}\right|\right) \rho(x) d x \\
& \quad \leq\left\|\bar{f}_{n}-\bar{f}\right\|_{2, \rho, T}^{2}
\end{aligned}
$$

by Proposition 7.6.

\section{Time-Inhomogeneous Additive Functionals and Dirichlet Processes}

In this section, we will be concerned with conditions on $u$ under which the functional $X^{u}=\left\{X_{s, t}^{u} \equiv u\left(t, X_{t}\right)-u\left(s, X_{s}\right) ; 0 \leq s \leq t \leq T\right\}$ is a Dirichlet process in the sense of Föllmer.

Let $\rho \in \mathcal{R}_{I}$. For $s \in[0, T)$, we set $P_{s, \rho}(\cdot)=\int_{\mathbb{R}^{d}} P_{s, x}(\cdot) \rho^{2}(x) d x$. 
Definition 4.1 We say that a CAF $A$ of finite variation is locally finite (resp., squareintegrable) if for every $\eta \in C_{0}^{+}\left(Q_{T}\right)$,

$$
\int_{0}^{T} E_{s, \rho} \int_{0}^{T} \eta\left(t, X_{t}\right) d\left|A_{s, \cdot}\right|{ }_{t} d s<\infty \quad\left(\text { resp., } \int_{0}^{T} E_{s, \rho}\left|A_{s, \cdot}\right|_{T}^{2} d s<\infty\right) .
$$

Definition 4.2 (i) Let $(s, x) \in Q_{\hat{T}}$ and $r \in[s, T]$. We say that a $\left\{\mathcal{G}_{t}^{s}\right\}$-adapted process $Y$ is a continuous Dirichlet process on $[r, T]$ under $P_{s, x}$ if

$$
Y_{t}=M_{t}+A_{t}, \quad t \in[r, T], P_{s, x} \text {-a.s., }
$$

where $M$ is a continuous $\left(\left\{\mathcal{G}_{t}^{s}\right\}, P_{s, x}\right)$-square-integrable martingale on $[r, T]$ and $A$ is a continuous $\left\{\mathcal{G}_{t}^{s}\right\}$-adapted process on $[r, T]$ such that $\langle A\rangle_{r}^{T}=0$ in the sense of (3.3). We say that $Y$ is a continuous Dirichlet process on $(s, T]$ under $P_{s, x}$ if it is a continuous Dirichlet process on $[r, T]$ under $P_{s, x}$ for every $r \in(s, T]$.

(ii) Let $\left\{\Pi_{m}=\left\{t_{0}, t_{1}, \ldots, t_{i(m)}\right\}\right\}$ be a sequence of partitions of $[s, T]$ whose meshsize converges to zero as $m \rightarrow \infty$. If $Y$ admits decomposition of the form (4.1) with a continuous $\left\{\mathcal{G}_{t}^{S}\right\}$-adapted process $A$ on $[r, T]$ such that $\langle A\rangle_{r}^{T}=0$ along $\left\{\Pi_{m}\right\}$ then we call it a continuous Dirichlet process along $\left\{\Pi_{m}\right\}$.

Given $u \in \mathcal{W}_{\rho}$ set

$$
M_{s, t}^{u} \equiv \int_{s}^{t} \nabla u\left(\theta, X_{\theta}\right) d M_{s, \theta}=\int_{s}^{t} \sigma \nabla u\left(\theta, X_{\theta}\right) d B_{s, \theta}, \quad 0 \leq s \leq t \leq T,
$$

where $B$ is defined by (3.4).

Theorem 4.3 Assume that $u \in \mathcal{W}_{\rho}$. Then there exists a quasi-continuous version of $u$ (still denoted by $u$ ) such that

(i) For every $(s, x) \in Q_{\hat{T}}$ the functional $X^{u}$ is a continuous Dirichlet process on $(s, T]$ under $P_{s, x}$ with the decomposition

$$
X_{r, t}^{u}=M_{r, t}^{u}+A_{r, t}^{u}, \quad s<r \leq t \leq T, P_{s, x} \text {-a.s. }
$$

where

$$
A_{r, t}^{u}=\int_{r}^{t} f^{0}\left(\theta, X_{\theta}\right) d \theta+\int_{r}^{t} a^{-1} \bar{f}\left(\theta, X_{\theta}\right) d^{*} X_{\theta}, \quad s<r \leq t \leq T, P_{s, x} \text {-a.s. }
$$

with $f^{0}, \bar{f} \in \mathbb{L}_{2, \rho}\left(Q_{T}\right)$ such that $\mathcal{L} u=f^{0}+\operatorname{div} \bar{f}$, where $\mathcal{L}=\frac{\partial}{\partial t}+L_{t}$.

(ii) For q.e. $(s, x) \in Q_{\hat{T}}$ decomposition (4.3), (4.4) holds true with $r=s$.

(iii) If $\frac{\partial u}{\partial t} \in \mathbb{L}_{2, \rho}\left(Q_{T}\right)+\mathbb{L}_{p^{\prime}}\left(0, T ; W_{p^{\prime}, \rho}^{-1}\right)$ with $p>2$ then for q.e. $(s, x) \in Q_{\hat{T}}, X^{u}$ is a Dirichlet process on $[s, T]$ under $P_{s, x}$ with decomposition (4.3) for $r=s$.

(iv) For every sequence $\left\{\Pi_{m}\right\}$ of partitions of $[0, T]$ whose mesh-size converges to zero as $m \rightarrow \infty$ there exists a subsequence $\left\{\Pi_{m^{\prime}}\right\}$ such that $X^{u}$ is a continuous Dirichlet process on $[s, T]$ along $\left\{\Pi_{m^{\prime}}^{s, T}=\Pi_{m} \cap[s, T]\right\}$ for q.e. $(s, x) \in Q_{\hat{T}}$ admitting decomposition (4.3) for $r=s$. 
Proof (i) First, note that it is known that if $u \in \mathcal{W}_{\rho}$ then there exist $f^{0}, \bar{f} \in \mathbb{L}_{2, \rho}\left(Q_{T}\right)$ such that $\mathcal{L} u=f^{0}+\operatorname{div} \bar{f}$. Let us fix $(s, x) \in Q_{\hat{T}}$. Let $\Phi^{\varepsilon}=f_{\varepsilon}^{0}+\operatorname{div} \bar{f}_{\varepsilon}$, where $f_{\varepsilon}^{i}$, $i=0, \ldots, d$, are standard mollifications of $f^{i}$, and let $\bar{f}_{\varepsilon}=\left(f_{\varepsilon}^{1}, \ldots, f_{\varepsilon}^{d}\right)$. Let $u^{\varepsilon}(T)$ be the standard mollification of $u(T)$ and $u_{n}$ be a continuous version on $Q_{T}$ of a weak solution of the Cauchy problem

$$
\left(\frac{\partial}{\partial t}+L_{t}\right) u_{n}=\Phi^{n}, \quad u_{n}(T)=u^{n}(T),
$$

where $\Phi^{n}=\Phi^{\varepsilon}, u^{n}(T)=u^{\varepsilon}$ with $\varepsilon=1 / n$. From [33] we know that $P_{s, x}$-a.s.,

$$
X_{r, t}^{u_{n}}=M_{r, t}^{u_{n}}+A_{r, t}^{u_{n}},
$$

for $s \leq r \leq t \leq T$, where

$$
A_{r, t}^{u_{n}}=\int_{r}^{t}\left(f_{n}^{0}+\operatorname{div} \bar{f}_{n}\right)\left(\theta, X_{\theta}\right) d \theta
$$

Let us define a version of $u$ (still denoted by $u$ ) as follows: $u(s, x)=\lim _{n \rightarrow \infty} u_{n}(s, x)$ if the limit exists, and zero otherwise. It is known (see [21]) that $u_{n} \rightarrow u$ in $\mathcal{W}_{\rho}$. Next, let

$$
A_{r, t}^{u}=\int_{r}^{t} f^{0}\left(\theta, X_{\theta}\right) d \theta+\int_{r}^{t} a^{-1} \bar{f}\left(\theta, X_{\theta}\right) d^{*} X_{\theta}, \quad s \leq r \leq t \leq T .
$$

By Proposition 3.3 and (3.14), for every $(s, x) \in Q_{\hat{T}}$ and $r \in(s, T], X_{r, t}^{u_{n}} \rightarrow X_{r, t}^{u}$, $M_{r, t}^{u_{n}} \rightarrow M_{r, t}^{u}, A_{r, t}^{u_{n}} \rightarrow A_{r, t}^{u}$ in $\mathbb{L}_{1}\left(\Omega, P_{s, x}\right)$ uniformly in $t \in[r, T]$. Therefore, passing to the limit in (4.6), we get (4.3), (4.4). By (3.16), for every $(s, x) \in Q_{\hat{T}}$ and $r \in$ $(s, T]$,

$$
\begin{aligned}
& \left\langle\int_{r} a^{-1} \bar{f}\left(\theta, X_{\theta}\right) d^{*} X_{\theta}\right\rangle_{r}^{T} \\
& \quad=\left\langle\int_{r} a^{-1} \bar{f}\left(\theta, X_{\theta}\right) d M_{s, \theta}+\int_{T+s-.}^{T+s-r} a^{-1} \bar{f}\left(\bar{\theta}, \bar{X}_{\theta}\right) d N_{s, \theta}^{s, x}\right\rangle_{r}^{T}
\end{aligned}
$$

under $P_{s, x}$. Let $\left\{\bar{f}_{n}\right\} \subset C_{0}^{\infty}\left(Q_{T}\right)$ be a sequence such that $\bar{f}_{n} \rightarrow \bar{f}$ in $\mathbb{L}_{2, \rho}\left(Q_{T}\right)$. Then

$$
\begin{gathered}
E_{s, x} \sum_{t_{i} \in \Pi_{m}^{r, T}}\left|\int_{t_{1}}^{t_{2}}\left(\bar{f}-\bar{f}_{n}\right)\left(\theta, X_{\theta}\right) d M_{s, \theta}+\int_{T+s-t_{2}}^{T+s-t_{1}}\left(\bar{f}-\bar{f}_{n}\right)\left(\bar{\theta}, \bar{X}_{\theta}\right) d N_{s, \theta}^{s, x}\right|^{2} \\
\quad \leq C E_{s, x} \int_{r}^{T}\left|\bar{f}_{n}-\bar{f}\right|^{2}\left(\theta, X_{\theta}\right) d \theta \leq C \rho^{-2}(x)(r-s)^{-d / 2}\left\|\bar{f}_{n}-\bar{f}\right\|_{2, \rho, T} .
\end{gathered}
$$

From this and the fact that $\left\langle\int_{r}^{\cdot} \bar{f}_{n}\left(\theta, X_{\theta}\right) d^{*} X_{\theta}\right\rangle_{r}^{T}=0, r \in(s, T], P_{s, x}$-a.s. for every $(s, x) \in Q_{\hat{T}}$, we get the first assertion of (i). To prove (ii), it suffices to pass to the limit with $r \rightarrow s^{+}$in (4.3) (if the limit exists) and use Corollary 3.4. Since from (i) it follows that $\left\langle\int_{r}^{\cdot} \bar{f}\left(\theta, X_{\theta}\right) d^{*} X_{\theta}\right\rangle_{r}^{T}=0, r \in(s, T], P_{s, x}$-a.s. for every $(s, x) \in Q_{\hat{T}}$, 
to prove (iii), it suffices to show that for q.e. $(s, x) \in Q_{\hat{T}}$ there exists the covariation $\left\langle\int_{s}^{\cdot} a^{-1} \bar{f}\left(\theta, X_{\theta}\right) d^{*} X_{\theta}\right\rangle_{s}^{T}$ under $P_{s, x}$. But the last statement is a direct consequence of Proposition 3.9. Finally, to prove (iv), let us set $B=\left\{(s, x) \in Q_{T}\right.$ : $\left.\limsup _{m \rightarrow \infty} E_{s, x} \sum_{t_{i} \in \Pi_{m}}\left|A_{s, t_{i+1}}-A_{s, t_{i}}\right|^{2}>0\right\}$ and $\tau=\inf \left\{t \in[s, T):\left(t, X_{t}\right) \in K\right\}$, where $K$ is a compact subset such that $K \subset B$. Then by the strong Markov property with random shift and additivity of $A$,

$$
\begin{aligned}
P_{s, x}(\tau<\infty) & =P_{s, x}\left(\limsup _{m \rightarrow \infty} E_{\tau, X_{\tau}} \sum_{t_{i} \in \Pi_{m}}\left|A_{\tau, t_{i+1}}-A_{\tau, t_{i}}\right|^{2}>0, \tau<\infty\right) \\
& =P_{s, x}\left(\limsup _{m \rightarrow \infty} E_{s, x}\left(\sum_{t_{i} \in \Pi_{m}}\left|A_{\tau, t_{i+1}}-A_{\tau, t_{i}}\right|^{2} \mid \mathcal{G}_{\tau}^{s}\right)>0, \tau<\infty\right) \\
& \leq P_{s, x}\left(\limsup _{m \rightarrow \infty} E_{s, x}\left(\sum_{t_{i} \in \Pi_{n}}\left|A_{s, t_{i+1}}-A_{s, t_{i}}\right|^{2} \mid \mathcal{G}_{\tau \wedge T}^{s}\right)>0\right) .
\end{aligned}
$$

Set

$$
T_{m}(s, x, \omega)=E_{s, x}\left(\sum_{t_{i} \in \Pi_{m}}\left|A_{s, t_{i+1}}-A_{s, t_{i}}\right|^{2} \mid \mathcal{G}_{\tau \wedge T}^{s}\right)
$$

and define the measure $\Pi$ as in the proof of Proposition 3.5. Since we know already that $\left\langle A_{s,},\right\rangle_{s}^{T}=0$ under $P_{s, x}$ for a.e. $(s, x) \in Q_{T}$, it follows that $T_{n} \wedge M \rightarrow 0$ in $\mathbb{L}_{1}\left(Q_{T} \times \Omega, \Pi\right)$, and hence that there exists a subsequence (still denoted by $m$ ) such that $T_{m} \rightarrow 0, \Pi$-a.e. Therefore, $T_{m}(s, x) \rightarrow 0, P_{s, x}$-a.s. for a.e. $(s, x) \in Q_{T}$, which proves that $\operatorname{cap}_{L}(K)=0$, hence that $\operatorname{cap}_{L}(B)=0$ by Remark 2.5.

Corollary 4.4 For every $\Phi \in \mathbb{L}_{2}\left(0, T ; H_{\rho}^{-1}\right)$ there exists a unique $C A F$ A of zero quadratic variation such that

$$
A_{r, t}=\int_{r}^{t} f^{0}\left(\theta, X_{\theta}\right) d \theta+\int_{r}^{t} a^{-1} \bar{f}\left(\theta, X_{\theta}\right) d^{*} X_{\theta}, \quad s<r \leq t \leq T, P_{s, x} \text {-a.s. }
$$

for any decomposition of $\Phi$ of the form $\Phi=f^{0}+\operatorname{div} \bar{f}, f^{0}, \bar{f} \in \mathbb{L}_{2, \rho}\left(Q_{T}\right)$.

In the sequel, we write $\int_{r}^{t} \Phi\left(\theta, X_{\theta}\right) d \theta=A_{r, t}, s \leq r \leq t \leq T$, or $\Phi \sim A$, if $A$ is the CAF corresponding to $\Phi \in \mathbb{L}_{2}\left(0, T ; H_{\rho}^{-1}\right)$ in the sense of the above corollary.

Remark 4.5 From the linearity of the operator $L_{t}$, it follows immediately that the mapping $\mathcal{W}_{\rho} \ni u \rightarrow A^{u}$, where $A^{u}$ is the functional of Theorem 4.3, is linear.

It is worth mentioning that the decomposition (4.3) implies Fukushima's decomposition of $X^{u}$ into a martingale AF of finite energy and a CAF of zero energy (for related results for time-independent $u$, see [32]). To state the result, let us recall first the definition of energy of time-inhomogeneous additive functionals of $\mathbb{X}$ and its basic properties. 
Definition 4.6 Let $A, B$ be CAFs of $\mathbb{X}$. We define the mutual energy of $A$ and $B$ by

$$
e(A, B)=\lim _{h \rightarrow 0^{+}} \frac{1}{h} \int_{0}^{T-h} E_{s, \rho} A_{s, s+h} B_{s, s+h} d s
$$

(whenever the limit exists), and we put $e(A)=e(A, A)$.

One can check that the energy has the following properties:

(i) $e(A+B)=e(A)+e(B)+2 e(A, B) \leq 2(e(A)+e(B))$,

(ii) If $e(A)=0$ and $e(B)<\infty$, then $e(A, B)=0$ and $e(A+B)=e(B)$.

Lemma 4.7 If $\Phi \in \mathbb{L}_{2}\left(0, T ; H_{\rho}^{-1}\right)$ then

$$
e\left(\int \Phi\left(\theta, X_{\theta}\right) d \theta\right) \leq C\|\Phi\|_{*}^{2} .
$$

Proof Let $s \in(0, T), h \in(0, T-s)$ and let $u \in \mathcal{W}_{\rho}$ be a solution of $\operatorname{PDE}(0, \Phi)$ on $[s, s+h]$. By Theorem 4.3 and Remark 2.3, there exists a quasi-continuous version of $u$ (still denoted by $u$ ) such that for a.e. $x \in \mathbb{R}^{d}$,

$$
X_{s, t}^{u}=M_{s, t}^{u}+A_{s, t}^{u}, \quad t \in[s, s+h], P_{s, x} \text {-a.s., }
$$

where

$$
A_{s, t}^{u}=\int_{s}^{t} \Phi\left(\theta, X_{\theta}\right) d \theta, \quad t \in[s, s+h], P_{s, x} \text {-a.s. }
$$

Hence, by Proposition 7.6 and Theorem 7.1,

$$
\begin{aligned}
E_{s, \rho}\left|\int_{s}^{s+h} \Phi\left(\theta, X_{\theta}\right) d \theta\right|^{2} & \leq C\left(\|\nabla u\|_{2, \rho, s, s+h}^{2}+\sup _{s \leq t \leq s+h}\|u(t)\|_{2, \rho}^{2}\right) \\
& \leq C\left(\left\|f^{0}\right\|_{2, \rho, s, s+h}+\|\bar{f}\|_{2, \rho, s, s+h}\right),
\end{aligned}
$$

where $f^{0}, \bar{f} \in \mathbb{L}_{2, \rho}\left(Q_{T}\right)$ are such that $\Phi=f^{0}+\operatorname{div}(\bar{f})$. From the above inequality, the result easily follows.

Corollary 4.8 Let $M^{u}, A^{u}$ be AFs of the decomposition (4.3). Then $e\left(M^{u}\right)<\infty$, $e\left(A^{u}\right)=0$.

Proof Using (4.2) and Proposition 7.6, one can check that $e\left(M^{u}\right) \leq C\|\nabla u\|_{2, \rho, T}^{2}$ $<\infty$. To prove that $e\left(A^{u}\right)=0$, let us write $\mathcal{L} u=\Phi$ and define $\Phi^{n}, A^{u_{n}}$ as in the proof of Theorem 4.3. Since the CAF $A^{u_{n}}$ has finite variation, direct calculation shows that $e\left(A^{u_{n}}\right)=0$. From this, Lemma 4.7 and property (i), it follows that

$$
e\left(A^{u}\right) \leq 2 e\left(A^{u}-A^{u_{n}}\right) \leq C\left(\left\|f^{0}-f_{n}^{0}\right\|_{2, \rho, T}^{2}+\left\|\bar{f}-\bar{f}_{n}\right\|_{2, \rho, T}^{2}\right)
$$

for $n \in \mathbb{N}$, which completes the proof. 


\section{Continuous Additive Functionals of Zero-Quadratic Variation}

Given a CAF $A$, we set

$$
D(A)=\left\{(s, x) \in Q_{\hat{T}}: A_{s,}, \text { is continuous and additive on }[s, T] \text { under } P_{s, x}\right\}
$$

and

$$
D_{0}(A)=\left\{(s, x) \in D(A) ; E_{s, x} \int_{s}^{T}\left|A_{s, t}\right|^{2} d t<\infty\right\} .
$$

For instance, if $A_{s, t}=\int_{s}^{t} f\left(\theta, X_{\theta}\right) d \theta, 0 \leq s \leq t \leq T$, for some $f \in \mathbb{L}_{1, \rho}\left(Q_{T}\right)$ then $\left\{(s, x) \in Q_{\hat{T}} ; P_{s, x}\left(\int_{s}^{T}\left|f\left(t, X_{t}\right)\right| d t<\infty\right)=1\right\} \subset D(A)$, and if

$$
A_{s, t}=\int_{s}^{t} \bar{f}\left(\theta, X_{\theta}\right) d^{*} X_{\theta}, \quad 0 \leq s \leq t \leq T,
$$

for some $\bar{f} \in \mathbb{L}_{2, \rho}\left(Q_{T}\right)$ then $N^{c} \subset D(A)$, where $N$ is defined in Corollary 3.8.

Lemma 5.1 Let $\bar{f} \in \mathbb{L}_{2, \rho}\left(Q_{T}\right)$ and let $A$ be defined by (5.1). Then $\operatorname{cap}_{L}\left(\left(D_{0}(A)\right)^{c}\right)$ $=0$.

Proof Let $u \in \mathcal{W}_{\rho}$ be a solution of $\operatorname{PDE}(0, \Phi)$, where $\Phi=\operatorname{div}\left(a^{-1} \bar{f}\right)$. By Theorem 4.3 and Remark 2.3, there exists a quasi-continuous version of $u$ (still denoted by $u$ ) such that for every $s \in(0, T)$ and a.e. $x \in \mathbb{R}^{d}$,

$$
X_{s, t}^{u}=M_{s, t}^{u}+A_{s, t}^{u}, \quad s \leq t \leq T, P_{s, x} \text {-a.s. }
$$

where

$$
A_{s, t}^{u}=\int_{s}^{t} \bar{f}\left(\theta, X_{\theta}\right) d^{*} X_{\theta}, \quad s \leq t \leq T, P_{s, x} \text {-a.s. }
$$

Hence, by Proposition 7.6 and Theorem 7.1,

$$
E_{s, \rho} A_{s, t}^{2} \leq C\|\Phi\|_{*}, \quad 0<s \leq t \leq T .
$$

Consequently, $E_{s, x} \int_{s}^{T}\left|A_{s, t}\right|^{2} d t<\infty$ for a.e. $(s, x) \in Q_{\hat{T}}$, and hence for q.e. $(s, x) \in$ $Q_{\hat{T}}$ by Corollary 3.4 .

Let $\Phi \in \mathbb{L}_{2}\left(0, T ; H_{\rho}^{-1}\right), A \sim \Phi$, and let $f^{0}, \bar{f} \in \mathbb{L}_{2, \rho}\left(Q_{T}\right)$ be such that $\Phi=$ $f^{0}+\operatorname{div} \bar{f}$. Our next goal is to define the integral with respect to $A$ and show that $A$ is determined by its $\alpha$-potential.

Given $\eta \in \mathcal{W}_{\rho} \cap \mathcal{B}_{b}\left(Q_{T}\right)$, we set

$$
\begin{aligned}
(\eta \cdot A)_{r, t}= & \int_{r}^{t} \eta\left(\theta, X_{\theta}\right) d A_{s, \theta} \equiv \int_{r}^{t} \eta f^{0}\left(\theta, X_{\theta}\right) d \theta-\int_{r}^{t} \nabla \eta \bar{f}\left(\theta, X_{\theta}\right) d \theta \\
& +\int_{r}^{t} a^{-1} \bar{f} \eta\left(\theta, X_{\theta}\right) d^{*} X_{\theta}, \quad 0 \leq s<r \leq t \leq T
\end{aligned}
$$


Observe that from (3.14), (3.15) it follows that all the integrals on the right-hand side of (5.2) are well defined. Moreover, setting

$$
N_{2}=\left(D\left(\int \eta f^{0} d t\right)\right)^{c} \cup\left(D\left(\int \nabla \eta \bar{f} d t\right)\right)^{c} \cup\left(D\left(\int a^{-1} \bar{f} \eta d^{*} X\right)\right)^{c},
$$

we see that $\operatorname{cap}_{L}\left(N_{2}\right)=0$ and for every $(s, x) \in N_{2}^{c}$ the right-hand side of (5.2) converges $P_{s, x}$-a.s. to a finite limit as $r \rightarrow s^{+}$. Thus, (5.2) defines a CAF of $\mathbb{X}$.

From the following proposition, it follows in particular that $\eta \cdot A$ does not depend on the choice of $f^{0}, \bar{f}$ in the decomposition of $\Phi$.

Proposition 5.2 Let $\Phi \in \mathbb{L}_{2}\left(0, T, H_{\rho}^{-1}\right)$ and let $A \sim \Phi$.

(i) For every bounded $\eta \in \mathcal{W}_{\rho}$, there exists a sequence $\left\{A^{n}\right\}$ of locally finite CAFs of finite variation such that for q.e. $(s, x) \in Q_{\hat{T}}$,

$$
E_{s, x} \sup _{s \leq t \leq T}\left|\int_{s}^{t} \eta\left(\theta, X_{\theta}\right) d A_{s, \theta}^{n}-\int_{s}^{t} \eta\left(\theta, X_{\theta}\right) d A_{s, \theta}\right| \rightarrow 0 .
$$

(ii) There exists a sequence $\left\{A^{n}\right\}$ of locally finite CAFs of finite variation such that for every bounded $\eta \in \mathcal{W}_{\rho},(s, x) \in Q_{\hat{T}}$ and $r \in(s, T]$,

$$
E_{s, x} \sup _{r \leq t \leq T}\left|\int_{r}^{t} \eta\left(\theta, X_{\theta}\right) d A_{r, \theta}^{n}-\int_{r}^{t} \eta\left(\theta, X_{\theta}\right) d A_{r, \theta}\right| \rightarrow 0 .
$$

Proof Let $A^{n}=A^{u_{n}}$, where $A^{u_{n}}$ is defined as in the proof of Theorem 4.3. Then the second part follows immediately from the definition of $\eta \cdot A^{n}, \eta \cdot A$ and (3.14), (3.15). To prove the first part, let us observe that by Proposition 7.6,

$$
\int_{Q_{T}}\left(E_{s, x} \sup _{s \leq t \leq T}\left|\left(\eta \cdot A^{n}\right)_{s, t}-(\eta \cdot A)_{s, t}\right|\right) \rho(x) d x \rightarrow 0,
$$

so the result follows from Proposition 3.3.

Remark 5.3 Notice that from Proposition 5.2 it follows that if $A$ is a CAF of finite variation corresponding to some $\Phi \in \mathbb{L}_{2}\left(0, T ; H_{\rho}^{-1}\right)$ then the usual LebesgueStieltjes integral $\int_{s}^{\cdot} \eta\left(t, X_{t}\right) d A_{s, t}$ and the integral in the sense of (5.2) coincide.

Using the definition (5.2) of the integral with respect to additive functionals of zero-quadratic variation, we can define the Laplace transform of such an additive functional.

For $\alpha>0$, we put

$$
U_{A}^{\alpha}(s, x)=E_{s, x} \int_{s}^{T} e^{-\alpha(t-s)} d A_{s, t}, \quad(s, x) \in D\left(e^{-(\cdot-s)} \cdot A\right)
$$

and

$$
U_{A}^{\alpha} \eta(s, x)=E_{s, x} \int_{s}^{T} e^{-\alpha(t-s)} \eta\left(t, X_{t}\right) d A_{s, t}, \quad(s, x) \in D\left(e^{-(\cdot-s)} \eta \cdot A\right)
$$


for $\eta \in \mathcal{W}_{\rho} \cap C_{b}\left(Q_{T}\right)$. In case $A$ is a CAF of finite variation, the integral in (5.3) is the usual Lebesgue-Stieltjes integral which is well defined for all $\eta \in C_{b}\left(Q_{T}\right)$.

In the sequel, we denote $D\left(e^{-(\cdot-s)} \eta \cdot A\right)$ by $D\left(U_{A}^{\alpha} \eta\right)$. If $A_{s, t}=t-s$, then we denote $U_{A}^{\alpha} \eta$ by $U^{\alpha} \eta$.

Let $\left\{R_{\alpha} ; \alpha \geq 0\right\}$ denote the resolvent of $\mathcal{L}$ on $\mathbb{L}_{2, \rho}\left(Q_{T}\right)$. Notice that if $\xi \in \mathcal{B}_{b}\left(Q_{T}\right)$ then $R_{\alpha} \xi \in \mathcal{W}_{\rho} \cap \mathcal{B}_{b}\left(Q_{T}\right)$ and $\nabla R_{\alpha} \xi \in \mathcal{B}_{b}\left(Q_{T}\right)$. Indeed, the first assertion follows immediately from the fact that $R_{\alpha} \xi$ is a strong solution of the Cauchy problem $(\alpha+\mathcal{L}) u=-\xi, u(T)=0$ and the representation formula

$$
R_{\alpha} \xi(s, x)=E_{s, x} \int_{s}^{T} e^{-\alpha(\theta-s)} \xi\left(\theta, X_{\theta}\right) d \theta, \quad(s, x) \in Q_{\hat{T}} .
$$

The second assertion follows from the formula

$$
\nabla R_{\alpha} \xi(s, x)=\int_{Q_{s T}} e^{-\alpha(\theta-s)} \xi(\theta, y) \nabla_{x} p(s, x, \theta, y) d \theta d y, \quad(s, x) \in Q_{\hat{T}}
$$

and integrability of $\nabla_{x} p(s, x, \cdot, \cdot)$ proven in [1, Theorem 10].

Proposition 5.4 Let A be CAF associated with some $\Phi \in \mathbb{L}_{2}\left(0, T ; H_{\rho}^{-1}\right)$. Then

$$
D_{0}(A) \subset \bigcap_{\xi \in \mathcal{B}_{b}\left(Q_{T}\right), \alpha, \beta \geq 0} D_{0}\left(e^{-\alpha(\cdot-s)} \cdot R_{\beta}(\xi) \cdot A\right) .
$$

Proof Let $\eta \in R_{\beta}\left(\mathcal{B}_{b}\left(Q_{T}\right)\right)$ and $\xi \in \mathcal{B}_{b}\left(Q_{T}\right)$ be such that $\eta=R_{\beta} \xi$. By Proposition 5.2, for every $(s, x) \in Q_{\hat{T}}$ and $r \in(s, T]$,

$$
E_{S, x} \sup _{r \leq t \leq T}\left|\int_{r}^{t} e^{-\alpha(\theta-s)} \eta\left(\theta, X_{\theta}\right) d A_{r, \theta}^{n}-\int_{r}^{t} e^{-\alpha(\theta-s)} \eta\left(\theta, X_{\theta}\right) d A_{r, \theta}\right| \rightarrow 0
$$

for some sequence $\left\{A^{n}\right\}$ of CAFs of finite variation. By the results proved in [33] and elementary calculations, for every $(s, x) \in Q_{\hat{T}}$ we have

$$
\begin{aligned}
\eta\left(t, X_{t}\right)= & \int_{t}^{T} e^{-\beta(\theta-s)} \xi\left(\theta, X_{\theta}\right) d \theta \\
& -\int_{t}^{T} e^{-\beta(\theta-s)} \sigma \nabla \eta\left(\theta, X_{\theta}\right) d B_{s, \theta}, \quad t \in[s, T], P_{s, x} \text {-a.s. }
\end{aligned}
$$

Hence applying the integration by parts formula to $A_{r, \cdot}^{n}\left(e^{-\alpha(\cdot-s)} \eta(\cdot, X).\right)$ and letting $n \rightarrow \infty$, we conclude that for every $(s, x) \in Q_{\hat{T}}$ under the measure $P_{s, x}$,

$$
\begin{aligned}
& \int_{r}^{t} e^{-\alpha(\theta-s)} \eta\left(\theta, X_{\theta}\right) d A_{r, \theta} \\
& \quad=\int_{r}^{t} e^{-(\alpha+\beta)(\theta-s)} \xi\left(\theta, X_{\theta}\right) A_{r, \theta} d \theta-\int_{r}^{t} e^{-(\alpha+\beta)(\theta-s)} \nabla \eta\left(\theta, X_{\theta}\right) A_{r, \theta} d B_{s, \theta} \\
& \quad-\alpha \int_{r}^{t} e^{-(\alpha+\beta)(\theta-s)} \eta\left(\theta, X_{\theta}\right) A_{r, \theta} d \theta+\eta\left(t, X_{t}\right) e^{-\alpha(t-s)} A_{r, t}
\end{aligned}
$$


for $s<r \leq t \leq T$. Since $\eta, \nabla \eta \in \mathcal{B}_{b}\left(Q_{T}\right)$ and $r \mapsto \eta\left(r, X_{r}\right)$ is continuous, letting $r \rightarrow s^{+}$we get (5.4) for $(s, x) \in D(A)$. From (5.4) with $r=s$, the proposition easily follows.

Proposition 5.5 Let $A, D$ be CAFs associated with some functionals in $\mathbb{L}_{2}\left(0, T, H_{\rho}^{-1}\right)$ such that $U_{D}^{\alpha} \eta=U_{A}^{\alpha} \eta$ on $D_{0}\left(U_{D}^{\alpha} \eta\right) \cap D_{0}\left(U_{A}^{\alpha} \eta\right)$ for every $\alpha>0$ and $\eta \in \mathcal{W}_{\rho} \cap C_{c}\left(Q_{T}\right)$. Then $A=D$.

Proof Without lost of generality, we may assume that $U_{D}^{\alpha} \eta=U_{A}^{\alpha} \eta$ on $D\left(U_{D}^{\alpha} \eta\right) \cap$ $D\left(U_{A}^{\alpha} \eta\right)$ for every $\eta \in \mathcal{W}_{\rho} \cap C_{b}\left(Q_{T}\right)$ because we can consider functionals $f \cdot A, f \cdot D$ with $f \in \mathcal{W}_{\rho} \cap C_{c}\left(Q_{T}\right)$ and from the equality $f \cdot A=f \cdot D$ for every such $f$ one can deduce that $A=D$. First, we show that

$$
U_{A}^{\alpha}\left(U^{\alpha} \eta\right)(s, x)=E_{s, x} \int_{s}^{T} e^{-\alpha(t-s)} \eta\left(t, X_{t}\right) A_{s, t} d t, \quad(s, x) \in D_{0}(A) .
$$

It is well known (see [21]) that $U^{\alpha} \eta \in \mathcal{W}_{\rho} \cap C_{b}\left(Q_{T}\right)$, so the above equality makes sense. Using the Markov property, Proposition 5.2 and Fubini's theorem, we have that for every $(s, x) \in Q_{\hat{T}}$ and $r \in(s, T]$

$$
\begin{aligned}
& E_{S, x} \int_{r}^{T} e^{-\alpha(t-s)} U^{\alpha} \eta\left(t, X_{t}\right) d A_{r, t} \\
& =\lim _{n \rightarrow \infty} E_{s, x} \int_{r}^{T} e^{-\alpha(t-s)} U^{\alpha} \eta\left(t, X_{t}\right) d A_{r, t}^{n} \\
& =\lim _{n \rightarrow \infty} E_{S, x} \int_{r}^{T} e^{-\alpha(t-s)}\left(E_{t, X_{t}} \int_{t}^{T} e^{-\alpha(\theta-t)} \eta\left(\theta, X_{\theta}\right) d \theta\right) d A_{r, t}^{n} \\
& =\lim _{n \rightarrow \infty} E_{s, x} \int_{r}^{T} e^{-\alpha(t-s)} E_{s, x}\left(\int_{t}^{T} e^{-\alpha(\theta-t)} \eta\left(\theta, X_{\theta}\right) d \theta \mid \mathcal{G}_{t}^{s}\right) d A_{r, t}^{n} \\
& =\lim _{n \rightarrow \infty} E_{s, x} \int_{r}^{T} e^{-\alpha(t-s)} \eta\left(t, X_{t}\right) A_{r, t}^{n} d t=E_{s, x} \int_{r}^{T} e^{-\alpha(t-s)} \eta\left(t, X_{t}\right) A_{r, t} d t
\end{aligned}
$$

Passing to the limit with $r \rightarrow s^{+}$for every $(s, x) \in D_{0}(A)$, we get that

$$
U_{A}^{\alpha}\left(U^{\alpha} \eta\right)(s, x)=E_{s, x} \int_{s}^{T} e^{-\alpha(t-s)} \eta\left(t, X_{t}\right) A_{s, t} d t, \quad(s, x) \in D_{0}(A) .
$$

By the above and the assumptions, it follows that

$$
\begin{aligned}
& E_{s, x} \int_{s}^{T} e^{-\alpha(t-s)} \eta\left(t, X_{t}\right) A_{s, t} d t=E_{s, x} \int_{s}^{T} e^{-\alpha(t-s)} \eta\left(t, X_{t}\right) D_{s, t} d t, \\
& \quad(s, x) \in D_{0}(A) .
\end{aligned}
$$


Hence

$$
E_{s, x} \eta\left(t, X_{t}\right) A_{s, t}=E_{s, x} \eta\left(t, X_{t}\right) D_{s, t}, \quad t \in[s, T]
$$

for $(s, x) \in D_{0}(A)$ by the well known properties of the Laplace transform. Consequently, using the Markov property and additivity of $A, D$ for every $0 \leq s \leq s^{\prime} \leq t^{\prime} \leq$ $t \leq T$ we have

$$
\begin{aligned}
E_{s, x} \eta\left(t^{\prime}, X_{t^{\prime}}\right) A_{s^{\prime}, t} & =E_{s, x} \eta\left(t^{\prime}, X_{t^{\prime}}\right) A_{s^{\prime}, t^{\prime}}+E_{s, x} \eta\left(t^{\prime}, X_{t^{\prime}}\right) A_{t^{\prime}, t} \\
& =E_{s, x}\left(E_{s^{\prime}, X_{s^{\prime}}}\left(\eta\left(t^{\prime}, X_{t^{\prime}}\right) A_{s^{\prime}, t^{\prime}}\right)\right)+E_{s, x}\left(\eta\left(t^{\prime}, X_{t^{\prime}}\right) E_{t^{\prime}, X_{t^{\prime}}} A_{t^{\prime}, t}\right) \\
& =E_{s, x}\left(E_{s^{\prime}, X_{s^{\prime}}}\left(\eta\left(t^{\prime}, X_{t^{\prime}}\right) D_{s^{\prime}, t^{\prime}}\right)\right)+E_{s, x}\left(\eta\left(t^{\prime}, X_{t^{\prime}}\right) E_{t^{\prime}, X_{t^{\prime}}}\left(D_{t^{\prime}, t}\right)\right) \\
& =E_{s, x} \eta\left(t^{\prime}, X_{t^{\prime}}\right) D_{s^{\prime}, t} .
\end{aligned}
$$

By induction, we get

$$
E_{s, x} \prod_{i=1}^{k} \eta\left(t_{i}, X_{t_{i}}\right) A_{t^{\prime}, t}=E_{S, x} \prod_{i=1}^{k} \eta\left(t_{i}, X_{t_{i}}\right) D_{t^{\prime}, t}
$$

for $0 \leq s \leq t^{\prime} \leq t_{1} \leq \cdots \leq t_{k} \leq t \leq T$ from which the lemma follows.

\section{The Semimartingale Structure of Additive Functionals}

In this section, we proceed with the study of the structure of the functional $X^{u}$. We will be concerned with additional conditions on $u \in \mathcal{W}_{\rho}$ under which $X^{u}$ is a semimartingale.

Let $S^{c}$ denote the set of all positive measures on $Q_{T}$ such that $\mu_{\mid \check{Q}_{T}} \ll \overline{\text { cap }}$ and $\mu\left(\{0\} \times \mathbb{R}^{d}\right)=\mu\left(\{T\} \times \mathbb{R}^{d}\right)=0$, and let $S_{0}^{c}$ be the set of measures $\mu \in S^{c}$ for which there exists $\Phi \in \mathbb{L}_{2}\left(0, T ; H_{\rho}^{-1}\right)$ such that (2.1) holds for every $\eta \in C_{c}^{\infty}\left(Q_{T}\right)$. First, we assume that $\mathcal{L} u \equiv \frac{\partial u}{\partial t}+L_{t} u \in S_{0}^{c}-S_{0}^{c}$ and then we consider the case where $\mathcal{L} u \in \mathcal{M}$. Of course, the first assumption implies the second one, but in general the converse implication is not true (see, e.g., [19, Example I.1]). Let us also remark that in general the functional $\mathcal{L} u$ is not a measure. For instance, if $d=1$, and $\mathcal{L} u=f^{\prime}$, then $\mathcal{L} u$ is a measure iff $f$ is locally of finite variation (see, e.g., [2, Proposition 3.6]). Finally, it is worth noting that the first assumption on the decomposition of $\mathcal{L} u$ appears naturally when considering obstacle problems (see, e.g., [25] and references therein).

Proposition 6.1 Assume that $u \in \mathcal{W}_{\rho}, \mathcal{L} u \in S_{0}^{c}-S_{0}^{c}$. Then there exist a quasicontinuous version of $u$ (still denoted by $u$ ) and square-integrable positive CAFs $C, R$ such that for every $(s, x) \in Q_{\hat{T}}$,

$$
\begin{aligned}
X_{r, t}^{u} & =M_{r, t}^{u}+C_{r, t}-R_{r, t}, \quad 0 \leq s<r \leq t \leq T, P_{s, x} \text {-a.s. } \\
E_{s, x}\left|C_{r, T}\right|^{2} \leq C \frac{\rho^{2}(x)}{(r-s)^{d / 2}}\left\|\mu_{1}\right\|_{*}, & E_{s, x}\left|R_{r, T}\right|^{2} \leq C \frac{\rho^{2}(x)}{(r-s)^{d / 2}}\left\|\mu_{2}\right\|_{*}
\end{aligned}
$$


and

$$
\begin{aligned}
& E_{s, x} \int_{r}^{T} \xi\left(t, X_{t}\right) d C_{r, t}=\int_{Q_{r T}} \xi(t, y) p(s, x, t, y) d \mu_{1}(t, y), \\
& E_{s, x} \int_{r}^{T} \xi\left(t, X_{t}\right) d R_{r, t}=\int_{Q_{r T}} \xi(t, y) p(s, x, t, y) d \mu_{2}(t, y)
\end{aligned}
$$

for all $\xi \in C_{0}\left(Q_{T}\right)$, where $\mu_{1}, \mu_{2} \in S_{0}^{c}$ are such that $\mathcal{L} u=\mu_{1}-\mu_{2}$. Moreover, for q.e. $(s, x) \in Q_{\hat{T}}$, (6.1), (6.3), (6.4) hold with $r=s$.

Proof By Theorem 4.3, there exists a CAF $A^{u}$ such that (4.3) holds. We are going to show that $A^{u}$ is a CAF of finite variation. Since $\mu_{1}, \mu_{2} \in S_{0}^{c}$, there exist $f^{0}, g^{0}, \bar{f}, \bar{g} \in$ $\mathbb{L}_{2, \rho}\left(Q_{T}\right)$ such that $\mu_{1}=f^{0}+\operatorname{div} \bar{f}, \mu_{2}=g^{0}+\operatorname{div} \bar{g}$. Let $f_{n}^{0}, g_{n}^{0}, \bar{f}_{n}, \bar{g}_{n}$ denote the standard mollifications of $f^{0}, g^{0}, \bar{f}, \bar{g}$, respectively, and let $\mu_{1}^{n}=f_{n}^{0}+\operatorname{div} \bar{f}_{n}$, $\mu_{2}^{n}=g_{n}^{0}+\operatorname{div} \bar{g}_{n}$. It is clear that $\mu_{1}^{n}, \mu_{2}^{n}$ are positive and $\mu_{1}^{n}, \mu_{2}^{n} \in \mathbb{L}_{2, \rho}\left(Q_{T}\right)$. Set

$$
C_{s, t}^{n}=\int_{s}^{t}\left(f_{n}^{0}+\operatorname{div} \bar{f}_{n}\right)\left(\theta, X_{\theta}\right) d \theta, \quad R_{s, t}^{n}=\int_{s}^{t}\left(g_{n}^{0}+\operatorname{div} \bar{g}_{n}\right)\left(\theta, X_{\theta}\right) d \theta,
$$

for $0 \leq s \leq t \leq T$ and

$$
\begin{aligned}
& C_{r, t}=\int_{r}^{t} f^{0}\left(\theta, X_{\theta}\right) d \theta+\int_{r}^{t} a^{-1} \bar{f}\left(\theta, X_{\theta}\right) d^{*} X_{\theta}, \\
& R_{r, t}=\int_{r}^{t} g^{0}\left(\theta, X_{\theta}\right) d \theta+\int_{r}^{t} a^{-1} \bar{g}\left(\theta, X_{\theta}\right) d^{*} X_{\theta}
\end{aligned}
$$

for $0 \leq s<r \leq t \leq T$. It is clear that for every $(s, x) \in Q_{\hat{T}}$,

$$
A_{r, t}^{u}=C_{r, t}-R_{r, t}, \quad 0 \leq s<r \leq t \leq T, P_{s, x} \text {-a.s. }
$$

By (3.14), (3.15), for every $(s, x) \in Q_{\hat{T}}$ and $r \in(s, T]$,

$$
E_{S, x} \sup _{r \leq t \leq T}\left(\left|C_{r, t}^{n}-C_{r, t}\right|+\left|R_{r, t}^{n}-R_{r, t}\right|\right) \rightarrow 0,
$$

which implies (6.1). Now, let $v \in \mathcal{W}_{\rho}$ be such that $\mathcal{L} v=\mu_{1}$ and $v(T)=0$. By (6.1), there exists a CAF $\tilde{C}$ such that $X^{v}=M^{v}+\tilde{C}$ in the sense of (6.1). Since $C, \tilde{C}$ satisfy (6.5), $C=\tilde{C}$. Hence, by Aronson's upper estimate and a priori estimates for PDEs,

$$
\begin{aligned}
E_{s, x}\left|C_{r, T}\right|^{2} & \leq C\left(E_{s, x}\left|M_{r, T}^{v}\right|^{2}+E_{s, x}\left|v\left(r, X_{r}\right)\right|^{2}\right) \\
& \leq C \frac{\rho^{2}(x)}{(r-s)^{d / 2}}\left(\|\nabla v\|_{2, \rho, T}^{2}+\sup _{0 \leq t \leq T}\|v(t)\|_{2, \rho}^{2}\right) \leq C \frac{\rho^{2}(x)}{(r-s)^{d / 2}}\left\|\mu_{1}\right\|_{*},
\end{aligned}
$$

which proves (6.2). To show (6.3), (6.4) let us fix $(s, x) \in Q_{\hat{T}}, r \in(s, T]$ and choose $\xi \in C_{0}^{\infty}\left(Q_{T}\right)$ so that $\xi \mathbf{1}_{Q_{s+\delta}}=0$ for some $\delta \in(0, T-s)$. Then, by Proposition 7.4, 
$\eta=\xi p(s, x, \cdot, \cdot) \in \mathbb{L}_{2}\left(0, T ; H_{\rho}^{1}\right)$ and

$$
\begin{aligned}
E_{s, x} \int_{s+\delta}^{T} \xi\left(t, X_{t}\right) d C_{s, t} & =\lim _{n \rightarrow \infty} E_{s, x} \int_{s+\delta}^{T} \xi\left(t, X_{t}\right) d C_{s, t}^{n} \\
& =\lim _{n \rightarrow \infty} \Phi_{1}^{n}(\eta)=\Phi_{1}(\eta)=\int_{s+\delta}^{T} \int_{\mathbb{R}^{d}} \xi(t, y) p(s, x, t, y) d \mu_{1}
\end{aligned}
$$

by (6.2), (6.7) and the fact that $\mu_{1}^{n} \rightarrow \mu_{1}$ in $\mathbb{L}_{2}\left(0, T ; H_{\rho}^{-1}\right)$. From this we easily get (6.3) and (6.4). Passing to the limit with $r \rightarrow s^{+}$in (6.1) and using (6.5), (6.6) and Corollary 3.4, we get (6.1) with $r=s$ for q.e. $(s, x) \in Q_{\hat{T}}$. Similarly, passing to the limit with $r \rightarrow s^{+}$in (6.3) and (6.4) and using (2.2) we get (6.3) and (6.4) with $r=s$ for q.e. $(s, x) \in Q_{\hat{T}}$.

From now on, we write $C \sim \mu$ if the CAF $C$ is associated with the measure $\mu$ in the sense of (6.3). From the above theorems, we get in particular the well known Revuz correspondence for smooth measures. However, in the case of the diffusion $\left(X, P_{s, x}\right)$, this correspondence may be expressed via density of the process which we present in the following corollary.

Remark 6.2 Repeating proofs of Lemmas 2.2.8 and 2.2.9 in [18], one can show that if $\mu \in S^{c}$ then there exists a sequence $\left\{F_{n}\right\}$ (called nest) of closed subsets of $\check{Q}_{T}$ such that $\mu\left(\check{Q}_{T} \backslash \bigcup_{n=1}^{+\infty} F_{n}\right)=0, \lim _{n \rightarrow \infty} \overline{\operatorname{cap}}\left(K-F_{n}\right)=0$ for every compact $K \subset \check{Q}_{T}$ and $\mathbf{1}_{F_{n}} d \mu \in S_{0}^{c}$ for every $n \in \mathbb{N}$.

Definition 6.3 We say that $d K: \Omega \times \mathcal{B}([0, T]) \rightarrow \mathbb{R}$ is a random measure if

(a) $d K(\omega)$ is a measure for every $\omega \in \Omega$,

(b) $\omega \mapsto d K(\omega)$ is $(\mathcal{G}, \mathcal{B}(\mathcal{M}[0, T]))$-measurable,

(c) $\int_{s}^{t} d K_{\theta}$ is $\mathcal{G}_{t}^{s}$-measurable for every $0 \leq s \leq t \leq T$.

Remark 6.4 By the results proven in [26], one can associate with the operator $\mathcal{L}$ a Hunt process $\left\{\left(Z_{t}, \tilde{P}_{z}\right), t \geq 0, z \in \mathbb{R}^{d+1}\right\}$. Actually, it follows from [26] that $\tilde{P}_{z}$ coincides with $P_{s, x}$ for $z=(s, x) \in Q_{\hat{T}}$ and that $Z_{t}=\left(\tau(t), X_{\tau(t)}\right)$, where $\tau$ is the uniform motion to the right, i.e., $\tau(t)=\tau(0)+t$ and $\tau(0)=s$ under $P_{s, x}$.

Lemma 6.5 Let $\left\{d K^{n}\right\}$ be a sequence of random measures. Assume that for $(s, x) \in F \subset Q_{\hat{T}}$ there exist random elements $d K^{s, x}:(\Omega, \mathcal{G}) \rightarrow\left(\mathcal{M}^{+}([0, T])\right.$, $\mathcal{B}\left(\mathcal{M}^{+}([0, T])\right)$ such that

$$
d K^{n}(\cdot, X .) \rightarrow d K^{s, x} \quad \text { in } \mathcal{M}^{+}([0, T]) \text { in probability } P_{s, x} .
$$

Then there exists a random measure $d K$ such that

$$
d K^{s, x}=d K, \quad P_{s, x} \text {-a.s. }
$$

for every $(s, x) \in F$. 
Proof Let $n_{0}(s, x)=0$ and let

$$
n_{k}(s, x)=\inf \left\{m>n_{k-1}(s, x), \sup _{p, q \geq m} P_{s, x}\left(d_{M}\left(d K^{p}, d K^{q}\right)>2^{-k}\right)<2^{-k}\right\}
$$

for $k \geq 1$. By induction, $n_{k} \in \mathcal{B}\left(Q_{\hat{T}}\right)$ for every $k \geq 0$, and hence $d L^{s, x, k}=d K^{n_{k}(s, x)}$ is $\mathcal{B}\left(Q_{\hat{T}}\right) \otimes \mathcal{G} / \mathcal{B}\left(\mathcal{M}^{+}([0, T])\right)$ measurable. Put

$$
d L^{s, x}(\omega)= \begin{cases}\lim _{k \rightarrow \infty} d L^{s, x, k}(\omega) \text { in } \mathcal{M}^{+}([0, T]) & \text { if the limit exists } \\ 0 & \text { otherwise. }\end{cases}
$$

By the Borel-Cantelli lemma, for every $(s, x) \in F$ the limit in (6.8) exists $P_{s, x}$-a.s. and $d L^{s, x}=d K^{s, x}, P_{s, x}$-a.s. Putting $d K(\omega)=d L^{Z_{0}(\omega)}$, we get a random measure having the desired properties.

Let $\mu \in S^{c}$. In what follows, by $d \mu(\cdot, X$.) we denote a random measure such that for q.e. $(s, x) \in Q_{\hat{T}}$,

$$
E_{s, x} \int_{s}^{T} \xi\left(t, X_{t}\right) d \mu\left(t, X_{t}\right)=\int_{Q_{s T}} \xi(t, y) p(s, x, t, y) d \mu(t, y)
$$

for every $\xi \in \mathcal{B}^{+}\left(Q_{T}\right)$.

Corollary 6.6 For every $\mu \in S^{c}$ there exists a unique random measure $d \mu(\cdot, X$.). Moreover, for every $\mu \in S_{0}^{c}$ and $s \in[0, T)$,

$$
E_{s, \rho}\left(\int_{s}^{T} d \mu\left(t, X_{t}\right)\right)^{2} \leq C\|\mu\|_{*}^{2} .
$$

Proof Uniqueness follows from Proposition 5.5 and Remark 6.2. Let $\mu \in S_{0}^{c}$ and let $u$ be a unique solution of $\operatorname{PDE}(0, \mu)$. By Proposition 4.3, there exist a unique positive CAF $A^{\mu}$ such that $A^{\mu} \sim \mu$ and a version of $u$ (still denoted by $u$ ) such that for every $(s, x) \in Q_{\hat{T}}$ and $r \in(s, T]$,

$$
u\left(r, X_{r}\right)=A_{r, T}^{\mu}-M_{r, T}^{u}, \quad 0 \leq s<r \leq T, P_{s, x} \text {-a.s. }
$$

By Theorem 4.3, the random measures

$$
d K^{n}(\omega)= \begin{cases}\Phi_{n}\left(t, X_{t}(\omega)\right) d t & \text { if } \int_{0}^{T} \Phi_{n}\left(t, X_{t}(\omega)\right) d t<\infty \\ 0 & \text { otherwise }\end{cases}
$$

where $\Phi_{n}$ are defined as in the proof Theorem 4.3, satisfy the assumptions of Lemma 6.5. Hence there exists a unique random measure $d \mu(\cdot, X$.) such that $\int_{s}^{t} d \mu\left(\theta, X_{\theta}\right)=A_{s, t}^{\mu}, s \leq t \leq T, P_{s, x}$-a.s. for q.e. $(s, x) \in Q_{\hat{T}}$. Therefore, by (6.11),

$$
u\left(r, X_{r}\right)=\int_{r}^{T} d \mu\left(\theta, X_{\theta}\right)-M_{r, T}^{u}, \quad 0 \leq s<r \leq T, P_{s, x} \text {-a.s. }
$$


Integrating (6.12) with respect to $\rho^{2} d m$ and using Proposition 7.2 yields

$$
E_{s, \rho}\left(\int_{r}^{T} d \mu\left(\theta, X_{\theta}\right)\right)^{2} \leq C\left(\|u(r)\|_{2, \rho, T}^{2}+\|\nabla u\|_{2, \rho, T}^{2}\right) \leq C\|\mu\|_{*}^{2}
$$

for every $r \in(s, T]$, the last inequality being a consequence of Theorem 7.1. The result now follows from Fatou's lemma. Now, let $\mu \in S^{c}$. Then, by Remark 6.2, there exists a nest $\left\{F_{n}\right\}$ such that $\mu_{n}=\mathbf{1}_{F_{n}} d \mu \in S_{0}^{c}$. By what has already been proven, for each $n \in \mathbb{N}$ there exists the random measure $d \mu_{n}(\cdot, X$.). Let us observe that if $n \leq m$ then $\mathbf{1}_{F_{n}} d \mu_{m}=d \mu_{n}$, which implies that $\mathbf{1}_{F_{n}} d \mu_{m}(\cdot, X$. $)=d \mu_{n}(\cdot, X$. $)$. Therefore, $d \mu_{m}(\cdot, X$. $) \geq d \mu_{n}(\cdot, X$.). By Lemma 6.5 , it follows that there exists a random measure $d K$ such that $d K=\lim _{n \rightarrow \infty} d \mu_{n}\left(\cdot, X\right.$.) in $\mathcal{M}^{+}([0, T])$ in probability $P_{s, x}$ for q.e. $(s, x) \in Q_{\hat{T}}$. It is clear that $d K$ satisfies (6.9). Therefore, $d K=d \mu(\cdot, X$.).

Remark 6.7 Let $u$ satisfy the assumptions of Proposition 6.1. Then by (6.1) and a priori estimates for BSDEs (see [13]), for every $(s, x) \in Q_{\hat{T}}$ and $r \in(s, T]$,

$$
\begin{aligned}
& E_{s, x} \sup _{r \leq t \leq T}\left|u\left(t, X_{t}\right)\right|^{2}+E_{s, x} \int_{r}^{T}|\nabla u|^{2}\left(\theta, X_{\theta}\right) d \theta \\
& \quad \leq C\left(E_{s, x}\left|u\left(T, X_{T}\right)\right|^{2}+E_{s, x}\left(\int_{r}^{T} d \mu_{1}\left(\theta, X_{\theta}\right)\right)^{2}+E_{s, x}\left(\int_{r}^{T} d \mu_{2}\left(\theta, X_{\theta}\right)\right)^{2}\right) .
\end{aligned}
$$

Hence, if $E_{s, x}\left(\int_{r}^{T} d \mu_{1}\left(\theta, X_{\theta}\right)\right)^{2}+E_{s, x}\left(\int_{r}^{T} d \mu_{2}\left(\theta, X_{\theta}\right)\right)^{2}<\infty$, then (6.1), (6.3), (6.4) are satisfied with $r=s$. Consequently, by Corollary 6.6, for each fixed $s \in$ $[0, T),(6.1),(6.3),(6.4)$ are satisfied for a.e. $x \in \mathbb{R}^{d}$. If $s \in(0, T)$, this also follows from the fact that $\operatorname{cap}_{L}(\{s\} \times B)>0$ for every $B \in \mathcal{B}\left(Q_{T}\right)$ such that $m(B)>0$.

Definition 6.8 We say that $X^{u}$ is a locally finite semimartingale if it is a semimartingale under $P_{s, x}$ for q.e. $(s, x) \in Q_{T}$ and its finite variation part is a locally finite CAF.

Let us remark that the class of locally finite semimartingales appears naturally when considering Revuz duality for additive functionals (see [17]).

The next theorem shows that the condition $\mathcal{L} u \in \mathcal{M}$ is necessary and sufficient for $X^{u}$ to be a locally finite semimartingale.

Theorem 6.9 Let $u \in \mathcal{W}_{\rho}$.

(i) $\mathcal{L} u \in \mathcal{M}$ iff $X^{u}$ is a locally finite semimartingale.

(ii) Assume that $\mathcal{L} u \in \mathcal{M}$. Let $\mu=\mathcal{L} u$ and let $A^{u}$ denote the finite variation part of $X^{u}$. Then $d A^{u}=d \mu(\cdot, X$.).

Proof Suppose that $\mathcal{L} u \in \mathcal{M}$ and let $\mu=\mathcal{L} u$. From Theorem 2.2, it follows that $\mu \ll \overline{\text { cap. }}$. Let $\mu=\mu^{+}-\mu^{-}$be the canonical decomposition. Of course, $\mu^{+} \mu^{-} \ll$ $\overline{\text { cap. }}$. Hence, by Theorem 2.1, there exist $\gamma_{1}, \gamma_{2} \in S_{0}^{c}$ and $\alpha_{1}, \alpha_{2} \in \mathbb{L}_{1, \text { loc }}^{+}\left(Q_{T}\right)$ such 
that $\mu^{+}=\alpha_{1} d \gamma_{1}, \mu^{-}=\alpha_{2} d \gamma_{2}$. Put

$$
D_{s, t}^{u}=\int_{s}^{t} \alpha_{1}\left(\theta, X_{\theta}\right) d \gamma_{1}\left(\theta, X_{\theta}\right)-\int_{s}^{t} \alpha_{2}\left(\theta, X_{\theta}\right) d \gamma_{2}\left(\theta, X_{\theta}\right), \quad 0 \leq s \leq t \leq T .
$$

By Aronson estimates, for every $\eta \in C_{0}\left(Q_{T}\right)$,

$$
\int_{Q_{T}}\left(E_{s, x} \int_{s}^{T} \eta \alpha_{i}\left(t, X_{t}\right) d \gamma_{i}\left(t, X_{t}\right)\right) \leq C \int_{Q_{T}} \eta \alpha_{i} d \gamma_{i}, \quad i=1,2 .
$$

From the above and Proposition 3.3, it follows that for q.e. $(s, x) \in Q_{\hat{T}}$ the functional $D^{u}$ is well defined and $E_{s, x} \int_{s}^{T} \eta \alpha_{i}\left(t, X_{t}\right) d \gamma_{i}\left(t, X_{t}\right)<\infty$ for every $\eta \in C_{0}\left(Q_{T}\right)$. By Theorem 4.3,

$$
X_{s, t}^{u}=M_{s, t}^{u}+A_{s, t}^{u}, \quad t \in[s, T], P_{s, x} \text {-a.s. }
$$

for q.e. $(s, x) \in Q_{\hat{T}}$ with $A^{u}, M^{u}$ as in Theorem 4.3. We shall show that $A^{u}=D^{u}$. In view of Proposition 5.5, to prove this it suffices to show that for every $\eta \in \mathcal{W}_{\rho} \cap$ $C_{c}\left(Q_{T}\right)$,

$$
E_{s, x} \int_{s}^{T} \eta\left(t, X_{t}\right) d A_{s, t}^{u}=E_{s, x} \int_{s}^{T} \eta\left(t, X_{t}\right) d D_{s, t}^{u}
$$

for $(s, x) \in D_{0}\left(\eta \cdot A^{u}\right) \cap D_{0}\left(\eta \cdot D^{u}\right)$. Given $\delta \in(0, T-s)$, write

$$
L_{\delta}=E_{s, x} \int_{s+\delta}^{T} \eta\left(t, X_{t}\right) d A_{s, t}^{u}, \quad R_{\delta}=E_{s, x} \int_{s+\delta}^{T} \eta\left(t, X_{t}\right) d D_{s, t}^{u} .
$$

Then by Theorem 4.3,

$$
\begin{aligned}
L_{\delta}= & \int_{Q_{s+\delta, T}} \eta f^{0}(t, y) p(s, x, t, y) d t d y-\int_{Q_{s+\delta, T}} \nabla \eta \bar{f}(t, y) p(s, x, t, y) d t d y \\
& +\int_{Q_{s+\delta, T}} \eta \bar{f}(t, y) \nabla_{y} p(s, x, t, y) d t d y
\end{aligned}
$$

and

$$
\begin{aligned}
R_{\delta}= & \int_{Q_{s+\delta, T}} \alpha_{1} \eta(t, y) p(s, x, t, y) d \gamma_{1}(t, y) \\
& -\int_{Q_{s+\delta, T}} \alpha_{2} \eta(t, y) p(s, x, t, y) d \gamma_{2}(t, y) .
\end{aligned}
$$

Since $\eta p(s, x, \cdot, \cdot) \in C_{0}\left(Q_{s+\delta, T}\right) \cap \mathbb{L}_{2}\left(s+\delta, T ; H_{\rho}^{1}\right)$, it follows from the assumption that $L_{\delta}=R_{\delta}$ for every $\delta>0$. Letting $\delta \rightarrow 0^{+}$for $(s, x) \in D_{0}\left(\eta \cdot A^{u}\right) \cap D_{0}\left(\eta \cdot D^{u}\right)$, we get $L_{0}=R_{0}$.

Now, assume that $X^{u}$ is a locally finite semimartingale. Without loss of generality, we may and will assume that $b=0$. The general case can be handled easily by using Girsanov's theorem because under the change of measure removing the drift 
term in the decomposition of $X^{u}$ new terms of finite variation appear (see, e.g., [34, Sect. 4] for details). Then $A^{u}$ from the decomposition of $X^{u}$ of Theorem 4.3 is of finite variation. Given $\eta \in C_{c}\left(Q_{T}\right)$, put

$$
\mu(\eta)=\int_{\mathbb{R}^{d}}\left(E_{0, x} \int_{0}^{T} \eta\left(\theta, X_{\theta}\right) d A_{0, \theta}\right) .
$$

By our assumptions, the above integral is well defined and the functional $\mu$ is continuous with respect to the uniform convergence on compacts, which implies that $\mu$ is a measure. We shall show that $\mathcal{L} u=\mu$. Let $\eta \in R_{\alpha}\left(C_{c}^{\infty}\left(Q_{T}\right)\right) \subset D(\mathcal{L}) \subset \mathcal{W}_{\rho}$, where $R_{\alpha}$ is the resolvent of $\mathcal{L}$. Then, by Theorem 4.3,

$$
\eta\left(t, X_{t}\right)=\eta\left(T, X_{T}\right)-\int_{t}^{T} \mathcal{L} \eta\left(\theta, X_{\theta}\right) d \theta-\int_{t}^{T} \sigma \nabla \eta\left(\theta, X_{\theta}\right) d B_{s, \theta}, \quad t \in[s, T]
$$

$P_{s, x}$-a.s. for q.e. $(s, x) \in Q_{\hat{T}}$. Integrating by parts, we get

$$
\begin{aligned}
E_{0, x} u\left(0, X_{0}\right) \eta\left(0, X_{0}\right)= & E_{0, x} u\left(T, X_{T}\right) \eta\left(T, X_{T}\right)-E_{0, x} \int_{0}^{T} u\left(t, X_{t}\right) \mathcal{L} \eta\left(t, X_{t}\right) d t \\
& -E_{0, x} \int_{0}^{T} \eta\left(t, X_{t}\right) d A_{0, t}-E_{0, x} \int_{0}^{T}\langle a \nabla \eta, \nabla u\rangle\left(t, X_{t}\right) d t .
\end{aligned}
$$

Notice that $R_{\alpha} \xi \rho^{-2} \in \mathbb{L}_{2}\left(Q_{T}\right)$ if $\xi \in C_{c}^{\infty}\left(Q_{T}\right)$. This follows from Proposition 7.2 and the fact that

$$
R_{\alpha} \xi(s, x)=E_{s, x} \int_{0}^{T} \mathbf{1}_{[0, T]}(s+t) e^{-\alpha t} \xi\left(s+t, X_{s+t}\right) d t
$$

(see, e.g., [27]). Integrating (6.15) with respect to $x$ and using symmetry of the operator $L_{t}$, we get

$$
\langle u(0), \eta(0)\rangle_{2}=\langle u(T), \eta(T)\rangle_{2}-\left\langle u, \frac{\partial \eta}{\partial t}\right\rangle_{2, T}+\left\langle u, L_{t} \eta\right\rangle_{2, T}-\int_{Q_{T}} \eta d \mu,
$$

which proves that $\langle\mathcal{L} u, \eta\rangle_{2, T}=\int_{Q_{T}} \eta d \mu$ for all $\eta \in R_{\alpha}\left(C_{c}^{\infty}\left(Q_{T}\right)\right)$. That $\mathcal{L} u=\mu$ now follows from the strong continuity of the resolvent.

Using Theorem 6.1, one can prove a useful estimate for the first moment of the supremum of $X^{u}$ in terms of the norm of $u$ in $\mathcal{W}_{\rho}$.

Corollary 6.10 If $u \in \mathcal{W}_{\rho}$ then there is a quasi-continuous version of $u$ (still denoted by $u$ ) such that for every $s \in(0, T)$

$$
E_{s, \sqrt{\rho}} \sup _{s \leq t \leq T}\left|u\left(t, X_{t}\right)\right| \leq C\|u\|_{\mathcal{W}_{\rho}} .
$$


Proof Since $\partial u / \partial t \in \mathbb{L}_{2}\left(0, T ; H_{\rho}^{-1}\right), u$ admits the representation

$$
\frac{\partial u}{\partial t}+L_{t} u=f^{0}+\operatorname{div}(\bar{f})+\frac{1}{2} \operatorname{div}(a \nabla u)+b \nabla u
$$

for some $f^{0}, \bar{f} \in \mathbb{L}_{2, \rho}\left(Q_{T}\right)$. From Theorem 4.3, it follows that there exists a quasicontinuous version of $h$ (still denoted by $h$ ) such that

$$
\begin{aligned}
X_{s, t}^{u}= & M_{s, t}^{u}+\int_{s}^{t} f^{0}\left(\theta, X_{\theta}\right) d \theta+\int_{s}^{t} a^{-1} \bar{f}\left(\theta, X_{\theta}\right) d^{*} X_{\theta} \\
& +\frac{1}{2} \int_{s}^{t} \nabla u\left(\theta, X_{\theta}\right) d^{*} X_{\theta}+\int_{s}^{t} \nabla u\left(\theta, X_{\theta}\right) d \beta_{s, \theta}
\end{aligned}
$$

for q.e. $(s, x) \in Q_{\hat{T}}$. By Doob's $\mathbb{L}_{2}$-inequality,

$$
\begin{aligned}
E_{s, x} \sup _{s \leq t \leq T}\left|u\left(t, X_{t}\right)\right| \leq & C\left(E_{s, x}\left(\left|u\left(T, X_{T}\right)\right|^{2}+\int_{s}^{T}\left(\left|f^{0}\right|^{2}+|\nabla u|^{2}\right)\left(\theta, X_{\theta}\right) d \theta\right)\right)^{1 / 2} \\
& +\sup _{s \leq t \leq T}\left|\int_{t}^{T}\left(a^{-1} \bar{f}+\nabla u\right)\left(\theta, X_{\theta}\right) d^{*} X_{\theta}\right| .
\end{aligned}
$$

Multiplying the above inequality by $\rho$ and using Proposition 7.6, we obtain

$$
E_{s, \sqrt{\rho}} \sup _{s \leq t \leq T}\left|u\left(t, X_{t}\right)\right| \leq C\left(\|u(T)\|_{2, \rho}+\left\|f^{0}\right\|_{2, \rho, T}+\|\bar{f}\|_{2, \rho, T}+\|\nabla u\|_{2, \rho, T}\right) .
$$

Taking the infimum over all $f^{0}, \bar{f} \in \mathbb{L}_{2, \rho}\left(Q_{T}\right)$ such that (6.16) is satisfied yields

$$
E_{s, \sqrt{\rho}} \sup _{s \leq t \leq T}\left|u\left(t, X_{t}\right)\right| \leq C\left(\|u(T)\|_{2, \rho}+\left\|\frac{\partial u}{\partial t}\right\|_{*}+\|\nabla u\|_{2, \rho, T}\right) .
$$

This proves the desired estimate because the imbedding of $\mathcal{W}_{\rho}$ into the vector space $C\left([0, T], \mathbb{L}_{2, \rho}\left(\mathbb{R}^{d}\right)\right)$ is continuous (see, e.g., [23]).

To estimate the second moment of the supremum of $X^{u}$, we assume that $\mathcal{L} u \in$ $S_{0}^{c}-S_{0}^{c}$. It is worth noting that solutions of parabolic equations with the right-hand side in $\mathbb{L}_{2, \rho}\left(Q_{T}\right)$ and solutions of unilateral or bilateral problems satisfy that assumption.

Corollary 6.11 Let $u \in \mathcal{W}_{\rho}$ and $\mathcal{L} u \in S_{0}^{c}-S_{0}^{c}$. Then there is a quasi-continuous version of $u$ (still denoted by $u$ ) such that for every $s \in(0, T)$,

$$
E_{s, \rho} \sup _{s \leq t \leq T}\left|u\left(t, X_{t}\right)\right|^{2} \leq C\left(\left\|\mu^{+}\right\|_{*}^{2}+\left\|\mu^{-}\right\|_{*}^{2}\right)
$$

where $\mu^{+}, \mu^{-} \in S_{0}^{c}$ and $\mathcal{L} u=\mu^{+}-\mu^{-}$.

Proof By Theorem 6.1, $X^{u}$ admits the decomposition (6.1) for q.e. $(s, x) \in Q_{\hat{T}}$. Therefore, one can prove the desired estimate by the same method as in the proof of Corollary 6.10. 
Acknowledgement Research supported by the Polish Minister of Science and Higher Education under Grant N N201 372436.

Open Access This article is distributed under the terms of the Creative Commons Attribution Noncommercial License which permits any noncommercial use, distribution, and reproduction in any medium, provided the original author(s) and source are credited.

\section{Appendix}

For the convenience of the reader, we collect here some estimates for diffusions $\mathbb{X}$ associated with $L_{t}$ and related estimates on the fundamental solution $p$ of $L_{t}$ and weak solutions of the Cauchy problem

$$
\frac{\partial u}{\partial t}+L_{t} u=-\Phi, \quad u(T)=\varphi
$$

$\left(\operatorname{PDE}(\varphi, \Phi)\right.$ for short), where $\Phi \in \mathbb{L}_{2}\left(0, T ; H_{\rho}^{-1}\right)$. Recall that $u \in \mathcal{W}_{\rho}$ is a strong solution of $\operatorname{PDE}(\varphi, \Phi)$ if for any $\eta \in \mathbb{L}_{2}\left(0, T ; H_{\rho}^{1}\right)$,

$$
\begin{aligned}
\int_{t}^{T}\left\langle\frac{\partial u}{\partial s}(s), \eta(s)\right\rangle_{\rho} d s-\frac{1}{2} \int_{t}^{T}\left\langle a(s) \nabla u(s), \nabla\left(\rho^{2} \eta(s)\right)\right\rangle_{2} d s \\
\quad+\int_{t}^{T}\langle b(s) \nabla u(s), \eta(s)\rangle_{2, \rho, T} \\
=\int_{t}^{T}\left\langle f^{0}(s), \eta(s)\right\rangle_{2, \rho} d s-\int_{t}^{T}\left\langle\bar{f}(s), \nabla\left(\rho^{2} \eta(s)\right)\right\rangle_{2} d s
\end{aligned}
$$

for all $t \in[0, T]$, where $f^{0}, \bar{f} \in \mathbb{L}_{2, \rho}\left(Q_{T}\right)$ are such that $\Phi=f^{0}+\operatorname{div} \bar{f}$.

For the proof of the following theorem, see, e.g., [21, 23].

Theorem 7.1 For every $\Phi \in \mathbb{L}_{2}\left(0, T, H_{\rho}^{-1}\right)$ there exists a unique strong solution $u \in \mathcal{W}_{\rho}$ of $\operatorname{PDE}(\varphi, \Phi)$ and

$$
\sup _{0 \leq t \leq T}\|u(t)\|_{2, \rho}^{2}+\|\nabla u\|_{2, \rho, T}^{2} \leq C\left(\|\varphi\|_{2, \rho}^{2}+\|\Phi\|_{*}^{2}\right) .
$$

Proposition 7.2 Let $\rho \in \mathcal{R}$. There exist $0<C_{1} \leq C_{2}$ depending only on $\lambda, \Lambda, \Lambda_{1}, d$, $T$ and $\rho$ such that

$$
\begin{aligned}
C_{1} \int_{t}^{T} \int_{\mathbb{R}^{d}}|\psi(\theta, x)| \rho(x) d \theta d x & \leq \int_{t}^{T} \int_{\mathbb{R}^{d}} E_{s, x}\left|\psi\left(\theta, X_{\theta}\right)\right| \rho(x) d \theta d x \\
& \leq C_{2} \int_{t}^{T} \int_{\mathbb{R}^{d}}|\psi(\theta, x)| \rho(x) d \theta d x
\end{aligned}
$$

for any $\psi \in \mathbb{L}_{1, \rho}\left(Q_{T}\right)$ and $t \in[s, T]$. 
Proof Follows from Proposition 5.1 in Appendix in [3] because, by Aronson's estimates (see [1, Theorem 7]), there exist $c_{0}, c_{1}, c_{2}>0$ depending only on $\lambda, \Lambda, d, T$ such that for every $\theta \in(s, T]$,

$$
\begin{aligned}
c_{0} \int_{\mathbb{R}^{d}} E\left|\psi\left(\theta, x+X_{c_{1}(\theta-s)}\right)\right| \rho(x) d x & \leq \int_{\mathbb{R}^{d}} E_{s, x}\left|\psi\left(\theta, X_{\theta}\right)\right| \rho(x) d x \\
& \leq c_{2} \int_{\mathbb{R}^{d}} E\left|\psi\left(\theta, x+X_{c_{2}(\theta-s)}\right)\right| \rho(x) d x
\end{aligned}
$$

where $E$ denotes expectation with respect to the standard Wiener measure on $\Omega$.

We now provide useful estimates for moments of $\mathbb{X}$.

Lemma 7.3 For every $p \geq 1$ there is $C$ depending only on $\lambda, \Lambda, d, T$ and $p$ such that

$$
E_{s, x} \sup _{s \leq t \leq T}\left|X_{t}\right|^{p} \leq C(p)(1+|x|)^{p}
$$

Proof By [37], there exist $C_{1}, C_{2}>0$ such that for every $(s, x) \in Q_{\hat{T}}$ and $r \geq 0$,

$$
P_{s, x}\left(\sup _{s \leq t \leq T}\left|X_{t}-x\right|>r\right) \leq C_{1} \exp \left(\frac{-C_{2} r^{2}}{T-s}\right) .
$$

From this we conclude that for every $p \geq 0$

$$
E_{s, x} \sup _{s \leq t \leq T}\left|X_{t}-x\right|^{p} \leq C(p),
$$

from which the result follows.

The following estimates for $p$ and weak solutions of (7.1) are known, but originally stated in terms of $\mathbb{L}_{p, q, \rho}$-norms with $\rho \equiv 1$. At the expense of minor technical changes, their proofs my be adapted to the case of spaces with weight $\rho$ such that $\rho^{-1}$ is a polynomial. For the first proposition, see Theorems 5, 7 and 10; and for the second one, Theorems 5 and 10 in [1].

Proposition 7.4 Assume that $p, q \in(1,+\infty], \frac{d}{2 p}+\frac{1}{q}<1$. Then for any $(s, x) \in Q_{\hat{T}}$,

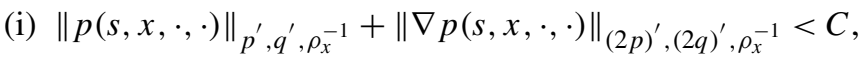

(ii) $p(s, x, \cdot, \cdot), \nabla p(s, x, \cdot, \cdot) \in \mathbb{L}_{2, \rho^{-1}}\left(Q_{s+\delta, T}\right)$ for every $\delta \in(0, T-s]$.

Proposition 7.5 Let $p, q$ satisfy the assumption of Proposition 7.4. Then there exists a continuous version $u$ of a weak solution of (7.1), and

$$
|u(t, x)| \leq C \rho^{-1}(x)\left(\|\varphi\|_{\infty, \rho}+\|f\|_{p, q, \rho}+\|\bar{f}\|_{2 p, 2 q, \rho}\right), \quad(t, x) \in Q_{\hat{T}} .
$$

Proposition 7.6 Assume that $p, q \in(1, \infty], \frac{d}{2 p}+\frac{1}{q}<\frac{1}{2}$. 
(i) For every $(s, x) \in Q_{\hat{T}}$ and $f \in \mathbb{L}_{p, q, \rho}\left(Q_{T}\right)$,

$$
E_{S, x} \int_{s}^{T}\left|f\left(t, X_{t}\right)\right|^{2} d t \leq C \rho^{-2}(x)\|f\|_{p, q, \rho} .
$$

(ii) For every $(s, x) \in Q_{\hat{T}}$ and $\bar{f} \in \mathbb{L}_{2 p, 2 q, \rho}\left(Q_{T}\right)$, the integral (3.7) is well defined and

$$
E_{S, x} \sup _{s \leq t \leq T}\left|\int_{s}^{t} \bar{f}\left(\theta, X_{\theta}\right) d^{*} X_{\theta}\right|^{2} \leq C \rho^{-2}(x)\|\bar{f}\|_{2 p, 2 q, \rho} .
$$

(iii) For every $s \in[0, T)$ and $f \in \mathbb{L}_{2, \rho}\left(Q_{T}\right)$,

$$
E_{s, \rho} \int_{s}^{T}\left|f\left(t, X_{t}\right)\right|^{2} d t \leq C\|f\|_{2, \rho, T} .
$$

(iv) If $\bar{f} \in \mathbb{L}_{2, \rho}\left(Q_{T}\right)$ then the integral (3.7) is well defined for a.e. $(s, x) \in Q_{\hat{T}}$,

$$
\int_{Q_{T}}\left(E_{s, x} \int_{s}^{T}\left|\bar{f}\left(t, X_{t}\right)\right| d\left|\alpha_{s, \cdot}^{s, x}\right|_{t}\right) \rho(x) d x d s \leq C\|\bar{f}\|_{2, \rho, T}
$$

and

$$
\int_{Q_{T}}\left(E_{s, x} \sup _{s \leq t \leq T}\left|\int_{s}^{t} \bar{f}\left(\theta, X_{\theta}\right) d^{*} X_{\theta}\right|\right) \rho(x) d x d s \leq C\|\bar{f}\|_{2, \rho, T} .
$$

Proof (i) Since $\rho^{-1}(x+y) \leq C \rho^{-1}(x) \rho^{-1}(y)$, applying Hölder's inequality gives

$$
\begin{aligned}
\int_{Q_{s T}}|f(t, y)|^{2} p(s, x, t, y) d t d y \leq & C \rho^{-2}(x) \int_{Q_{s T}}|f(t, y)|^{2} \rho^{2}(y) p(s, x, t, y) \\
& \times \rho_{x}^{-2}(y) d t d y \\
\leq & C \rho^{-2}(x)\|f\|_{p, q, \rho}^{2}\left\|p(s, x, \cdot, \cdot) \rho_{x}^{-2}\right\|_{(p / 2)^{\prime},(q / 2)^{\prime}},
\end{aligned}
$$

and the result follows from Proposition 7.4.

(ii) By (i), integrals with respect to backward and forward martingale are well defined. As for the finite variation part, observe that

$$
\begin{aligned}
E_{s, x} \int_{s}^{T}|\bar{f}|\left(\theta, X_{\theta}\right) d\left|\alpha_{s, \cdot}^{s, x}\right|_{\theta} & =\int_{Q_{s, T}}|\bar{f}(\theta, y)||\nabla p|(s, x, \theta, y) d \theta d y \\
& \leq C \rho^{-1}(x)\|\bar{f}\|_{2 p, 2 q, \rho}\|\nabla p(s, x, \cdot, \cdot)\|_{(2 p)^{\prime},(2 q)^{\prime}, \rho_{x}^{-1}}
\end{aligned}
$$

which is finite for every $(s, x) \in Q_{\hat{T}}$ by Proposition 7.4. Now, let $u$ be a weak solution of the Cauchy problem (7.1) with $\bar{f}=0, \varphi=0$. Then $E_{s, x} \int_{s}^{T}\left|\nabla u\left(\theta, X_{\theta}\right)\right|^{2} d \theta \leq$ $C \rho^{-2}(x)\|f\|_{2 p, 2 q, \rho}^{2}$ by [34, Theorem 4.1], and $|u(s, x)| \leq \rho^{-1}(x) C\|f\|_{2 p, 2 q, \rho}$ by 
Proposition 7.5. Moreover, from [34] we know that

$$
u\left(t, X_{t}\right)=\int_{t}^{T} a^{-1} \bar{f}\left(\theta, X_{\theta}\right) d^{*} X_{\theta}-\int_{t}^{T} \nabla u\left(\theta, X_{\theta}\right) d M_{s, \theta}, \quad t \in[s, T], P_{s, x} \text {-a.s. }
$$

By the above estimates and Doob's $\mathbb{L}_{2}$-inequality,

$$
\begin{aligned}
E_{s, x}\left|\int_{s}^{T} f\left(t, X_{t}\right) d^{*} X_{t}\right|^{2} & \leq C\left(E_{s, x} \sup _{s \leq t \leq T} \rho^{-2}\left(X_{t}\right)+\rho^{-2}(x)\right)\|f\|_{2 p, 2 q, \rho}^{2} \\
& \leq 2 C \rho^{-2}(x)\|f\|_{2 p, 2 q, \rho}^{2},
\end{aligned}
$$

the last inequality being a consequence of Corollary 7.3.

(iii) Follows from Proposition 7.2.

(iv) The fact that integrals with respect to backward and forward martingales are well defined follows directly from (iii). Modifying slightly [31, Lemma 5.2] to the case of time-inhomogeneous diffusions, we have for $\alpha>0$,

$$
\begin{aligned}
\mid E_{s, x} & \left.\int_{s}^{T}\left|\bar{f}\left(t, X_{t}\right)\right| d\left|\alpha_{s, \cdot}^{s, x}\right|_{t}\right|^{2} \\
\leq & \left|\int_{Q_{s} T}\right| f|| \frac{\partial p}{\partial y_{j}}|(s, x, t, y) d t d y|^{2} \\
\leq & C E_{s, x} \int_{s}^{T}(t-s)^{-\alpha}\left|\bar{f}\left(t, X_{t}\right)\right|^{2} d t \\
& \times \sum_{i, j=1}^{d} \int_{Q_{s} T}(t-s)^{\alpha} p^{-1} a_{i j} \frac{\partial p}{\partial y_{i}} \frac{\partial p}{\partial y_{j}}(s, x, t, y) d t d y \\
\leq & C E_{s, x} \int_{s}^{T}(t-s)^{-\alpha}\left|\bar{f}\left(t, X_{t}\right)\right|^{2} d t .
\end{aligned}
$$

Multiplying this inequality by $\rho$ and using the fact that the measure $\rho d m$ is finite on $\mathbb{R}^{d}$, we obtain by Jensen's inequality that

$$
\begin{aligned}
I & \equiv \int_{Q_{T}}\left(E_{s, x} \int_{s}^{T}\left|\bar{f}\left(t, X_{t}\right)\right| d\left|\alpha_{s, \cdot}^{s, x}\right|_{t}\right) \rho(x) d x d s \\
& \leq C\left(\int_{0}^{T} \int_{s}^{T}(t-s)^{-\alpha}\left(\int_{\mathbb{R}^{d}} E_{s, x}\left|f\left(t, X_{t}\right)\right|^{2} \rho^{2}(x) d x\right) d t d s\right)^{1 / 2} .
\end{aligned}
$$

Write $r(t)=\|f(t)\|_{2, \rho}^{2}$. From the above with $\alpha=1 / 2$ and (iii), we get

$$
\begin{aligned}
I^{2} & \leq C \int_{0}^{T} \int_{S}^{T}(t-s)^{-1 / 2} r(t) d t d s=C \int_{0}^{T}\left(\int_{0}^{t}(t-s)^{-1 / 2} d s\right) r(t) d t \\
& \leq C T^{1 / 2} \int_{0}^{T} r(t) d t=C T^{1 / 2}\|\bar{f}\|_{2, \rho, T}^{2},
\end{aligned}
$$


which proves the result. The second assertion is a direct consequence of (iii) and (7.2).

\section{References}

1. Aronson, D.G.: Non-negative solutions of linear parabolic equations. Ann. Sc. Norm. Super. Pisa 22, 607-693 (1968)

2. Ambrosio, L., Fusco, N., Pallara, D.: Functions of Bounded Variation and Free Discontinuity Problems. Oxford University Press, London (2000)

3. Bally, V., Matoussi, A.: Weak solutions for SPDEs and backward doubly stochastic differential equations. J. Theor. Probab. 14, 125-164 (2001)

4. Bertoin, J.: Les processus de Dirichlet et tant qu'espace de Banach. Stochastics 18, 155-168 (1986)

5. Biroli, M., Mosco, U.: Wiener estimates for parabolic obstacle problem. Nonlinear Anal. 11, 10051027 (1987)

6. Boccardo, L., Gallouët, T., Orsina, L.: Existence and uniqueness of entropy solutions for nonlinear elliptic equations with measure data. Ann. Inst. Henri Poincaré, Anal. Non Linéaire 13, 539-551 (1996)

7. Chitashvili, R., Mania, M.: On functions transforming Brownian motion into a Dirichlet process. In: Probability theory and mathematical statistics (Tokyo, 1995), pp. 20-27. World Sci. Publ., River Edge (1996)

8. Chitashvili, R., Mania, M.: On functions transforming a Wiener process into a semimartingale. Probab. Theory Relat. Fields 109(1997), 57-76 (1997)

9. Coquet, F., Mémin, J., Słomiński, L.: On non-continuous Dirichlet processes. J. Theor. Probab. 16, 197-216 (2003)

10. Dal Maso, G.: On the integral representation of certain local functionals. Ric. Mat. 22, 85-113 (1983)

11. Di Cristo, M., Kim, K., Nakamura, G.: (2009) Estimate of the fundamental solution for parabolic operators with discontinuous coefficients. arXiv:0906.4438v1

12. Dupoiron, K., Mathieu, P., San Martin, J.: Formule d'Itô pour des diffusions uniformement elliptiques, et processus de Dirichlet. Potential Anal. 21, 7-33 (2004)

13. El Karoui, N., Kapoudjian, C., Pardoux, E., Peng, S., Quenez, M.C.: Reflected solutions of backward SDEs, and related obstacle problems for PDE's. Ann. Probab. 25, 702-737 (1997)

14. Föllmer, H.: Dirichlet processes. In: Stochastic Integrals. Lecture Notes in Mathematics, vol. 851, pp. 476-478. Springer, Berlin (1981). Proc. Sympos., Univ. Durham, Durham (1980)

15. Föllmer, H., Protter, P. On Itô's formula for multidimensional Brownian motion. Probab. Theory Relat. Fields, 116, 1-20 (2000)

16. Föllmer, H., Protter, P., Shiryaev, A.N.: Quadratic covariation and an extension of Itô's formula. Bernoulli 1, 149-169 (1995)

17. Fukushima, M.: On semi-martingale characterizations of functionals of symmetric Markov processes. Electron. J. Probab. 4, 1-32 (1999)

18. Fukushima, M., Oshima, Y., Takeda, M.: Dirichlet Forms and Symmetric Markov Processes. De Gruyter Studies in Mathematics, vol. 19. Walter de Gruyter, New York (1994)

19. Hanouzet, B., Joly, J.L.: Méthodes d'ordre dans l'interprétation de certaines inéquations variationnelles et applications. J. Funct. Anal. 34, 217-249 (1997)

20. Klimsiak, T.: Strong solutions of semilinear parabolic equations with measure data and generalized backward stochastic differential equations. Potential Anal. (2011). doi:10.1007/s11118-011-9235-Z

21. Ladyzenskaya, O.A., Solonnikov, V.A., Ural'ceva, N.N.: Linear and Quasi-Linear Equations of Parabolic Type. Transl. Math. Monographs, vol. 23. Am. Math. Soc., Providence (1968)

22. Lejay, A.: A probabilistic representation of the solution of some quasi-linear PDE with a divergence form operator. Application to existence of weak solutions of FBSDE. Stoch. Process. Appl. 110, 145$176(2004)$

23. Lions, J.-L.: Quelques Methodes de Resolutions des Problemes aux Limites Non Lineaires. Dunod, Gauthier Villars, Paris (1968)

24. Lyons, T., Stoica, L.: The limits of stochastic integrals of differential forms. Ann. Probab. 27, 1-49 (1999)

25. Mokrane, A., Murat, F.: Proof of the Lewy-Stampacchia's inequality by a penalization method. Potential Anal. 9, 105-142 (1998) 
26. Oshima, Y.: On a construction of Markov processes associated with time dependent Dirichlet forms. Forum Math. 4, 395-415 (1992)

27. Oshima, Y.: Time-dependent Dirichlet forms and related stochastic calculus. Infin. Dimens. Anal. Quantum Probab. Relat. Top. 7, 281-316 (2004)

28. Pierre, M.: Parabolic capacity and Sobolev spaces. J. Math. Anal. 14, 522-533 (1983)

29. Rozkosz, A.: Stochastic representation of diffusions corresponding to divergence form operators. Stoch. Process. Appl. 63, 11-33 (1996)

30. Rozkosz, A.: A note on Dirichlet processes. Bull. Pol. Acad. Sci., Math. 48, 277-286 (2000)

31. Rozkosz, A.: On Dirichlet processes associated with second order divergence form operators. Potential Anal. 14, 123-148 (2001)

32. Rozkosz, A.: Time-inhomogeneous diffusions corresponding to symmetric divergence form operators. Probab. Math. Stat. 22, 231-252 (2002)

33. Rozkosz, A.: Backward SDEs and Cauchy problem for semilinear equations in divergence form. Probab. Theory Relat. Fields 125, 393-401 (2003)

34. Rozkosz, A.: On the Feynman-Kac representation for solutions of the Cauchy problem for parabolic equations in divergence form. Stochastics 77, 297-313 (2005)

35. Stannat, W.: The theory of generalized Dirichlet forms and its application in analysis and stochastics. Mem. Amer. Math. Soc. 142(678) (1999)

36. Stoica, I.L.: A Probabilistic interpretation of the divergence and BSDE's. Stoch. Process. Appl. 103, 31-55 (2003)

37. Stroock, D.W.: Diffusion semigroups corresponding to uniformly elliptic divergence form operators. Lect. Notes Math. 1321, 316-347 (1998) 